\title{
Characterising Equilibrium Selection in Global Games with Strategic Complementarities
}

\author{
Christian Basteck* \\ Tijmen R. Daniëls* \\ Frank Heinemann*
}

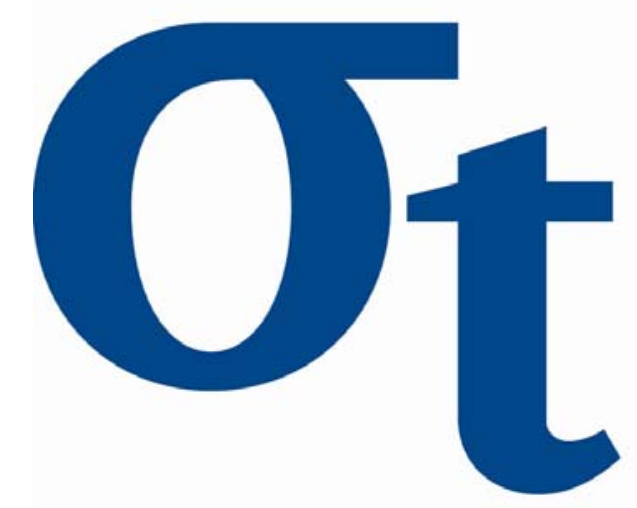




\title{
CHARACTERISING EQUILIBRIUM SELECTION IN GLOBAL GAMES WITH STRATEGIC COMPLEMENTARITIES
}

\author{
CHRISTIAN BASTECK TIJMEN R. DANIËLS* FRANK HEINEMANN
}

TECHNISCHE UNIVERSITÄT BERLIN

\begin{abstract}
Global games are widely used for equilibrium selection to predict behaviour in complete information games with strategic complementarities. We establish two results on the global game selection. First, we show that it is independent of the payoff functions of the global game embedding, though it may depend on the noise distribution. Second, we give a simple sufficient criterion for noise independence in many action games. A many action game may be noise independent if it can be suitably decomposed into smaller (say, binary action) games, for which there are simple criteria guaranteeing noise independence. We delineate the games where noise independence may be established by counting the number of players or actions. In addition, we give an elementary proof that robustness to incomplete information implies noise independence.
\end{abstract}

Keywords: global games, equilibrium selection, strategic complementarities.

JEL codes: $\mathrm{C} 72, \mathrm{D} 82$.

\section{Introduction}

Games with strategic complementarities typically have multiple equilibria that give rise to coordination problems. Economic applications cover a wide range of topics, including poverty traps and underdevelopment (see for example Ray [24]) or financial crises (see for example Diamond and Dybvig [9], Obstfeld [20]). A widely used approach to predict behaviour in such games is by embedding them in a "global game". A global game extends a complete information game $g$ by a payoff function $u$ that depends on an additional state parameter $\theta$ not directly observable by agents. The payoff function $u$ coincides with the payoff function $g$ at the true state, say $\theta^{*}$, but agents have to rely on noisy private signals about the true state. This leads to uncertainty not just about their own relevant payoff function, but also - and more importantly - about the beliefs of opponents.

Frankel, Morris and Pauzner [10] (henceforth "FMP") show that as the noise in private signals vanishes, agents coordinate on some action profile that is a Nash equilibrium of the complete information game $g$. This global game selection of $g$ may be used as an equilibrium prediction and to derive comparative statics results in games with multiple equilibria. Applications include Morris and Shin [17]; Cukierman, Goldstein and Spiegel

Date: First version: December 2009. This slightly revised version: January 2010.

*Corresponding author: tijmen.daniels@tu-berlin.de.

Support from Deutsche Forschungsgemeinschaft through SFB649 is gratefully acknowledged.

We thank Satoru Takahashi and participants at DGL09 for their helpful comments. 
[7]; Rochet and Vives [25]; Coresetti, Dasgupta, Morris and Shin [5]; Goldstein [11]; Corsetti, Guimarães and Roubini [6]; Guimarães and Morris [12], among others. The theory has also been corroborated by experimental evidence, see Heinemann, Nagel and Ockenfels [13] and [14].

Unfortunately, the global game selection in $g$ may depend on the fine details of the global game. A well known problem is that it may depend on the signals' noise distribution. FMP discuss the robustness of the global game selection with respect to the signals' noise distribution, and provide some conditions under which the global game selection is noise independent, that is, independent of this distribution.

In this paper, we establish two additional positive results on the robustness of global games. First, we show that the global game selection, though it may depend on the noise distribution, is always independent of the embedding payoff function $u$. This may come as a surprise, since the process of global game selection is often described informally as "infection" from high and low parameter regions.

Second, we provide new and simple conditions for noise independence in many-action games. We show that the global game selection in $g$ may be noise independent if $g$ can be suitably decomposed into smaller noise independent games. For example, we may split up a $n$-action game into many binary action games for which there are simple criteria that guarantee noise independence.

This approach is useful, since the simplest known criteria to establish noise independence are through counting the number of players and actions. Carlsson and Van Damme [4] show that two player, binary action games are noise independent. The global game selection is the risk dominant action profile. Morris and Shin [18] show how to find the global game selection in many player, symmetric, binary action games. Here, the global game selection is the best reply to the belief that the fraction of players choosing either action is uniformly distributed. Up to now, most applications of global games use these heuristics in binary action environments. Our result gives a simple tool to extend them to many action games.

But other criteria in terms of players and actions may also be applied. In this paper, we establish that all two player $2 \times n$ action games are noise independent. Oyama and Takahashi [22] show that two player, symmetric $3 \times 3$ games are noise independent. Conversely, FMP show that symmetric $4 \times 4$ games may not be noise independent. An example by Carlsson [3] shows that noise independence may fail in three player, binary action games. In this paper, we establish that it may also fail in two player, asymmetric $3 \times 3$ games. As far as we know, it is the first $3 \times 3$ example in the literature. It completes the characterisation of games where noise independence can be established simply by counting the number of players or actions.

Another useful criterion that guarantees the noise independence of $g$ is the existence of an equilibrium that is "robust to incomplete information", as defined by Kajii and Morris [15]. A heuristic argument may be found in Morris and Shin [18], but in this paper we give a formalisation. It allows one to make use of various known conditions 
for robustness to incomplete information when trying to determine whether some game is noise independent. Our result is closely related to a similar theorem of Oury and Tercieux, who use a somewhat stronger, slightly non-standard, notion of robustness to incomplete information to exploit a link with so-called "contagious" equilibria [21]. Instead, we use the standard notion and give a direct proof.

Our paper is organised as follows. Section 2 contains preliminary definitions and results. In section 3, we set out by giving a simple characterisation of the global game selection process. Instead of analysing the limit of a series of global games with shrinking noise, we show that a single incomplete information game with a fixed noise structure allows one to determine the global game selection in $g$. Moreover, this incomplete information game does not incorporate the whole range of the payoff function $u$ of the global game, but depends on the payoff structure of $g$ alone. Hence, it establishes that the global game selection in $g$ is independent of the embedding payoff function $u$. In section 4 we use this characterisation of the global game selection to establish our results on noise independence in many-action games. Section 5 contains the proof that robustness noise independence, based on our characterisation of the global game selection.

\section{Setting and Definitions}

In this paper we consider games with a finite set of players $I$, who have finite action sets $A_{i \in I}=\left\{0,1, \ldots, m_{i}\right\}$ which we endow with the natural ordering inherited from $\mathbb{N}$. We define the joint action space $A$ as $\prod_{i \in I} A_{i}$ and write $A_{-i}$ for $\prod_{j \neq i} A_{j}$. We say that $a=\left(a_{i}\right)_{i \in I} \in A$ is weakly greater than $a^{\prime}=\left(a_{i}^{\prime}\right)_{i \in I}$ if $a_{i} \geq a_{i}^{\prime}$ for all $i \in I$ and write $a \geq a^{\prime}$. A complete information game $g$ is specified by its real-valued payoff functions $g_{i \in I}\left(a_{i}, a_{-i}\right)$, where $a_{i}$ denotes $i$ 's action and $a_{-i}$ denotes the opposing action profile. A game $g$ is a game of strategic complementarities ${ }^{1}$ if greater opposing action profiles make greater actions more appealing, or more precisely, if for all $i, a_{i} \geq a_{i}^{\prime}, a_{-i} \geq a_{-i}^{\prime}$,

$$
g_{i}\left(a_{i}, a_{-i}\right)-g_{i}\left(a_{i}^{\prime}, a_{-i}\right) \geq g_{i}\left(a_{i}, a_{-i}^{\prime}\right)-g_{i}\left(a_{i}^{\prime}, a_{-i}^{\prime}\right) .
$$

\section{§2.1. Global Games}

Following FMP, we define a global game $G_{v}(u, \phi, f)$ as follows. It is an incomplete information game where payoffs depend on a real-valued random variable $\theta$, called the state parameter, which is distributed according to a continuous density $\phi$, called the prior distribution. For the most part, we will use $\theta$ to denote both the random variable and a specific value it assumes, but we denote the latter by $\theta^{*}$ if confusion could arise. The individual payoffs in the incomplete information game are given by $u_{i}\left(a_{i}, a_{-i}, \theta\right) . \quad f$ is a tuple of densities, whose support is a subset of $\left[-\frac{1}{2}, \frac{1}{2}\right]$, that we refer to as the noise structure. Each player $i \in I$ observes a private signal $x_{i}=\theta+v \eta_{i}$ about $\theta$, where $v>0$ is a scale factor and $\eta_{i}$ an error that is distributed according to the density $f_{i}$. We use

\footnotetext{
${ }^{1}$ Strictly speaking, it would be more correct to say that $g$ is a supermodular game [26, 27]. However, FMP use the term strategic complementarities in this context, so we stick to it.
} 
$x_{i}$ to denote both the random variable and the specific value it assumes, but sometimes denote the former by $X_{i}$ to avoid confusion. The random variables $\left\{\theta, \eta_{1}, \ldots, \eta_{I}\right\}$ are independently distributed.

Moreover, FMP define four conditions that the payoff function $u$ needs to fulfil.

A1 Strategic complementarities: For every value of $\theta$, the complete information game specified by $u_{i \in I}(\cdot, \theta)$ is a game of strategic complementarities.

A2 Dominance regions: Extreme values of $\theta$ make the extreme actions dominant choices. That is, there exist thresholds $\underline{\theta}<\bar{\theta}$ such that $[\underline{\theta}-v, \bar{\theta}+v]$ is contained in the interior of the support of $\phi$ and for all players $i$ and all opposing action profiles $a_{-i}$ we have

$$
u_{i}\left(0, a_{-i}, \theta\right)>u_{i}\left(a_{i}, a_{-i}, \theta\right) \text { for all } a_{i}>0 \text { and } \theta \leq \underline{\theta} \text {, }
$$

and

$$
u_{i}\left(m_{i}, a_{-i}, \theta\right)>u_{i}\left(a_{i}, a_{-i}, \theta\right) \text { for all } a_{i}<m_{i} \text { and } \theta \geq \bar{\theta} .
$$

A3 State monotonicity: Greater states make greater actions more appealing. More precisely, there exists $K>0$ such that for all $a_{i} \geq a_{i}^{\prime}$ and $\underline{\theta} \leq \theta^{\prime} \leq \theta \leq \bar{\theta}$ we have

$$
\begin{aligned}
& \left(u_{i}\left(a_{i}, a_{-i}, \theta\right)-u_{i}\left(a_{i}^{\prime}, a_{-i}, \theta\right)\right)-\left(u_{i}\left(a_{i}, a_{-i}, \theta^{\prime}\right)-u_{i}\left(a_{i}^{\prime}, a_{-i}, \theta^{\prime}\right)\right) \\
& \quad \geq K\left(a_{i}-a_{i}^{\prime}\right)\left(\theta-\theta^{\prime}\right) \geq 0 .
\end{aligned}
$$

A4 Payoff continuity: Each $u_{i}\left(a_{i}, a_{-i}, \theta\right)$ is continuous in the state parameter.

\section{§2.2. Strategies in Global Games}

A (pure) strategy for player $i$ is a (measurable) function $s_{i}: \mathbb{R} \rightarrow A_{i}$ and a (pure) strategy profile $s$ is a tuple of such strategies, $s=\left(s_{i}\right)_{i \in I}$. If $x$ is a tuple of signals $\left(x_{i}\right)_{i \in I}$, then $s(x)$ denotes the action profile $\left(s_{i}\left(x_{i}\right)\right)_{i \in I}$. Slightly abusing notation, for $x \in \mathbb{R}$ we also denote the action profile given by $\left(s_{i}(x)\right)_{i \in I}$ by $s(x)$. A strategy profile $s$ is increasing if each component $s_{i}$ is weakly increasing and left (right) continuous if each $s_{i}$ is left (right) continuous. We say that the strategy profile $s$ is weakly greater than the strategy profile $s^{\prime}$ if $s(x) \geq s^{\prime}(x)$ for all $x \in \mathbb{R}$ and write $s \geq s^{\prime}$.

The joint density of $X_{i}$ and $\theta$ is given by $f_{i}\left(\frac{x_{i}-\theta}{v}\right) \phi(\theta)$, so players can use Bayes rule to derive the conditional density of $\theta$ :

$$
f_{X_{i}=x_{i}}\left(\theta^{*}\right)=\frac{f_{i}\left(\frac{x_{i}-\theta^{*}}{v}\right) \phi\left(\theta^{*}\right)}{\int_{\mathbb{R}} f_{i}\left(\frac{x_{i}-\theta}{v}\right) \phi(\theta) d \theta} .
$$

Since all error terms are independent, the conditional density of $X_{-i}$ given $X_{i}=x_{i}$ can be calculated as

where

$$
\pi_{i}\left(x_{-i} \mid x_{i}\right):=\int_{\mathbb{R}} \pi_{-i}\left(x_{-i} \mid \theta\right) f_{X_{i}=x_{i}}(\theta) d \theta
$$

$$
\pi_{-i}\left(x_{-i} \mid \theta\right):=\prod_{j \neq i} f_{j}\left(\frac{x_{j}-\theta}{v}\right)
$$


denotes the conditional density of $X_{-i}$ given $\theta$. We will also use the notation

$$
\mathbb{P}\left(X_{-i} \in E \mid X_{i}=x_{i}\right):=\int_{E} \pi_{i}\left(x_{-i} \mid x_{i}\right) d x_{-i}
$$

to refer to probabilities conditional on the null set $\left\{X_{i}=x_{i}\right\}$. In the special case where $\theta$ is distributed uniformly, the conditional densities $\pi_{i}\left(x_{-i} \mid x_{i}\right)$ satisfy the following very useful symmetry property. (We give a derivation in the appendix.)

Lemma 1. If $\theta$ is uniformly distributed over the interval $[L, R]$, then we have, for any $x \in\left[L+\frac{1}{2}, R-\frac{1}{2}\right]^{I}$, and any $i, j \in I$, that $\pi_{i}\left(x_{-i} \mid x_{i}\right)=\pi_{j}\left(x_{-j} \mid x_{j}\right)$.

Given $x_{i}$ and assuming that opponents $j \neq i$ follow the strategies $s_{j}$ given by some strategy profile $s$, the action $a_{i} \in A_{i}$ yields an expected payoff of

$$
u_{i}\left(a_{i} \mid s, x_{i}\right):=\int_{\mathbb{R}} \int_{x_{-i} \in \mathbb{R}^{|I|-1}} u_{i}\left(a_{i}, s_{-i}\left(x_{-i}\right), \theta\right) \pi_{-i}\left(x_{-i} \mid \theta\right) d x_{-i} f_{X_{i}=x_{i}}(\theta) d \theta .
$$

against the induced opposing action distribution. Let $\beta(s)_{i}\left(x_{i}\right)$ denote the set of best replies of player $i$ conditional on the signal $x_{i}$, viz the set of actions that maximise the expected payoff:

$$
\beta(s)_{i}\left(x_{i}\right)=\underset{a_{i} \in A_{i}}{\arg \max } u_{i}\left(a_{i} \mid s, x_{i}\right) .
$$

A strategy profile $s$ is a (Bayes-Nash) equilibrium strategy profile, if it is a best reply to itself, i.e.

$$
\forall i \forall x_{i}, \quad s_{i}\left(x_{i}\right) \in \beta(s)_{i}\left(x_{i}\right) .
$$

The upper-best reply is defined as

$$
\hat{\beta}(s)_{i}\left(x_{i}\right)=\max \beta(s)_{i}\left(x_{i}\right)
$$

and determines the strategy profile $\hat{\beta}(s)$. The strategic complementarities imply that if one opposing action distribution dominates another, the upper-best reply to the former is weakly greater than to the latter. In particular, $\hat{\beta}$ is monotonic, i.e., $\hat{\beta}(s) \geq \hat{\beta}\left(s^{\prime}\right)$ if $s \geq s^{\prime}$ (see Topkis [26, 27] and Vives [28]). We can do upper-best reply iterations

$$
s, \hat{\beta}(s), \hat{\beta}(\hat{\beta}(s)), \hat{\beta}(\hat{\beta}(\hat{\beta}(s))), \ldots
$$

starting at some strategy profile $s$. If $\hat{\beta}(s)$ is weakly greater (smaller) than $s$, the resulting sequence of strategy profiles will be monotonically increasing (decreasing). As the action space is bounded, the resulting sequence will then converge pointwise to an equilibrium strategy profile. In particular, choosing the greatest strategy profile given by

$$
\forall i \forall x_{i}, \quad s_{i}^{0}\left(x_{i}\right)=m_{i},
$$

as a starting point, the best reply to the strategy profile $s^{0}$ can only be weakly smaller, so that the iteration will converge pointwise to the (necessarily) greatest equilibrium strategy profile (see figure 1).

As is usual when dealing with games of strategic complementarities, virtually all of our results are order-theoretic in nature. By standard order-theoretic duality, each result 


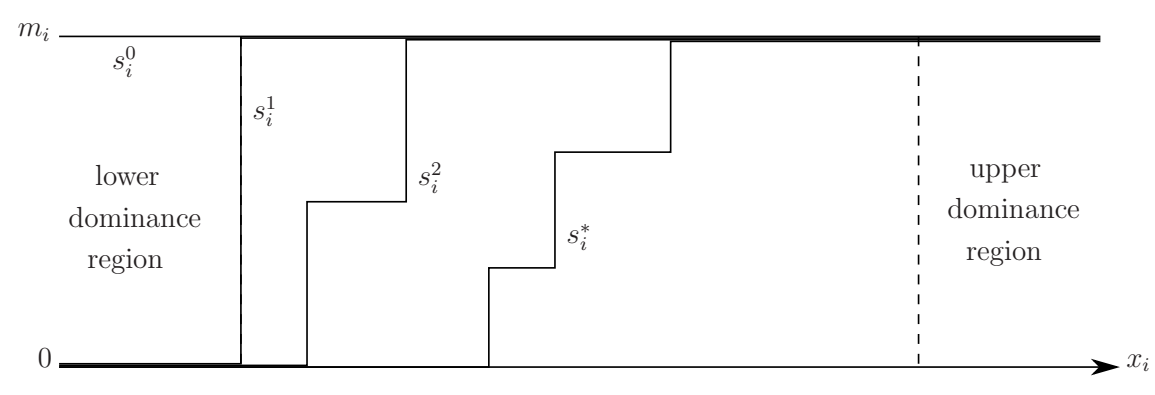

FIGURE 1. The strategy of player $i$ in an upper-best reply iteration leading to the greatest equilibrium strategy profile $s^{*}$.

implies a dual result with all order-theoretic notions reversed (see Davey and Priestley [8], p. 15). We will invoke this duality throughout the text.

\section{§2.3. Summary of FMP's Results}

FMP showed that in a global game the least and greatest equilibrium strategy profile converge to each other as the noise vanishes.

Theorem. (Theorem 1 in FMP) The global game $G_{v}(u, \phi, f)$ has an essentially unique equilibrium strategy profile in the limit as $v \rightarrow 0$. More precisely, there exists an increasing pure strategy profile $s^{f}$ such that if, for each $v>0, s_{v}$ is an equilibrium strategy profile of $G_{v}(u, \phi, f)$, then $\lim _{v \rightarrow 0} s_{v, i}\left(x_{i}\right)=s_{i}^{f}\left(x_{i}\right)$ for all $x_{i}$, except possibly at the finitely many discontinuities of $s^{f}$.

Moreover, FMP's proof implies that $s^{f}$ is independent of the prior distribution $\phi$ (cf. their lemma A3).

Since $s^{f}$ is determined up to its points of discontinuity, we will work with the left and right continuous versions of $s^{f}$, which we denote by $\underline{s}^{f}$ and $\bar{s}^{f}$ respectively.

To prove the theorem, FMP introduce the notion of a simplified global game $G_{v}(u, f)$ that differs from $G_{v}(u, \phi, f)$ in that $\theta$ is uniformly distributed over a large interval that contains $[\underline{\theta}-v, \bar{\theta}+v]$ and individual payoffs depend directly on the private signal $x_{i}$ rather than on the true state $\theta$. A simplified global game is much easier to analyse, as the uncertainty about the relevant individual payoff function vanishes, and for signals $x_{i}$ within $[\underline{\theta}, \bar{\theta}]$ the conditional densities of opponents' signals can be calculated easily as

$$
\pi_{i}\left(x_{-i} \mid x_{i}\right)=\int_{\mathbb{R}} \prod_{j \in I} f_{j}\left(\frac{x_{j}-\theta}{v}\right) d \theta .
$$

Theorem. (Lemma A1, A3, and A4 in FMP) The simplified global game $G_{v}(u, f)$ has an essentially unique, monotonically increasing equilibrium strategy profile $s_{v}^{f}$. In the limit as $v \rightarrow 0, s_{v}^{f}$ converges towards $s^{f}$ in horizontal distance, that is

for all $\varepsilon>0$ there is $\bar{v}>0$ such that for $v$ satisfying $0<v<\bar{v}$, we have:

$$
\forall i \forall x_{i}, \quad s_{v, i}^{f}\left(x_{i}+\varepsilon\right) \geq s_{i}^{f}\left(x_{i}\right) \geq s_{v, i}^{f}\left(x_{i}-\varepsilon\right)
$$

See figure 2. 


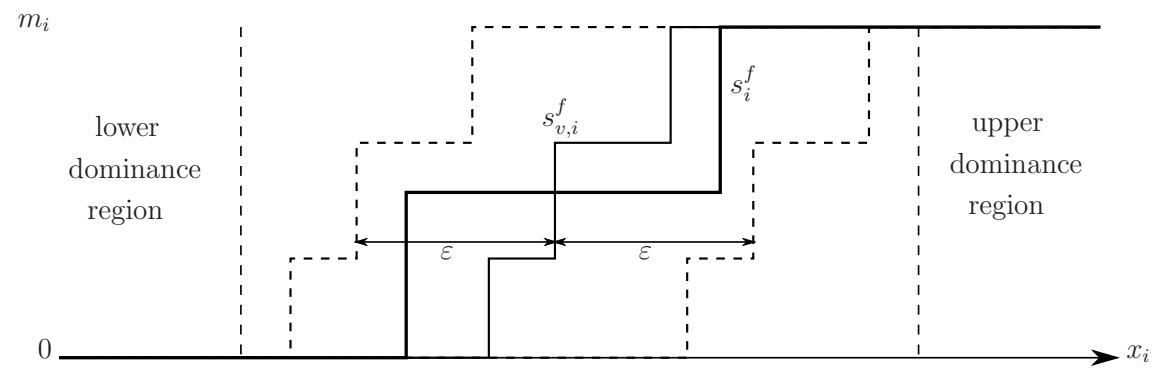

Figure 2. $s_{i}^{f}$ and $s_{v, i}^{f}$ for $v<\bar{v}$

\section{Equilibrium Selection in Global Games}

Let $g$ be a game of strategic complementarities. For a given global game $G_{v}(u, \phi, f)$, we define the embedded game $g\left(\theta^{*}\right)$ as the complete information game that has the same set of players and actions as the global game, and which has the payoff function given by $u\left(\cdot, \theta^{*}\right)$.

For $g\left(\theta^{*}\right)$, the global game can be viewed as an equilibrium refinement. The limit strategy profile of the global game, $s^{f}$, determines an action profile $s^{f}\left(\theta^{*}\right)$, which is in fact an equilibrium of the embedded game. Thus the global game approach generically selects a unique equilibrium of the game $g$, to which we refer as the global game selection (following Heinemann et al. [14]). In this section, we provide a conceptually simple characterisation of this selection process which shows that the selection depends only on the payoff structure of $g\left(\theta^{*}\right)$ and on the noise structure $f$.

\section{§3.1. Global Games as an Equilibrium Refinement}

Our first aim in this section is to show that this approach can by applied to any game with strategic complementarities. Let $G_{v}(u, \phi, f)$ be a given global game, and let $g$ be some game of strategic complementarities. We say that $G_{v}(u, \phi, f)$ embeds the complete information game $g$ if there is there is a $\theta^{*}$ such that $g=g\left(\theta^{*}\right)$.

Lemma 2. For any game of strategic complementarities $g$, there exists a global game $G_{v}(u, \phi, f)$ that embeds $g$.

We prove this by constructing a global game $G_{v}(u, \phi, f)$ such that $g=g(0)$.

Proof. Let $u$ be given by $u_{i}\left(a_{i}, a_{-i}, \theta\right):=g_{i}\left(a_{i}, a_{-i}\right)+\theta a_{i}$, for $i \in I, a_{i} \in A_{i}$ and $a_{-i} \in A_{-i}$. Clearly, each $u_{i}$ is continuous in $\theta$, so $u$ satisfies A4. For any fixed $\theta$ and $a_{i} \geq a_{i}^{\prime}, a_{-i} \geq a_{-i}^{\prime}$ we have

$$
\begin{aligned}
u_{i}\left(a_{i}, a_{-i}, \theta\right)-u_{i}\left(a_{i}^{\prime}, a_{-i}, \theta\right) & =g_{i}\left(a_{i}, a_{-i}\right)-g_{i}\left(a_{i}^{\prime}, a_{-i}\right)+\theta\left(a_{i}-a_{i}^{\prime}\right) \\
& \geq g_{i}\left(a_{i}, a_{-i}^{\prime}\right)-g_{i}\left(a_{i}^{\prime}, a_{-i}^{\prime}\right)+\theta\left(a_{i}-a_{i}^{\prime}\right) \\
& =u_{i}\left(a_{i}, a_{-i}^{\prime}, \theta\right)-u_{i}\left(a_{i}^{\prime}, a_{-i}^{\prime}, \theta\right),
\end{aligned}
$$


as $g$ is a game of strategic complementarities. So $u$ exhibits strategic complementarities as well and satisfies A1. For $a_{i} \geq a_{i}^{\prime}$ and $\theta \geq \theta^{\prime}$ we have that

$$
\begin{aligned}
& \left(u_{i}\left(a_{i}, a_{-i}, \theta\right)-u_{i}\left(a_{i}^{\prime}, a_{-i}, \theta\right)\right)-\left(u_{i}\left(a_{i}, a_{-i}, \theta^{\prime}\right)-u_{i}\left(a_{i}^{\prime}, a_{-i}, \theta^{\prime}\right)\right) \\
& \quad=g_{i}\left(a_{i}, a_{-i}\right)-g_{i}\left(a_{i}^{\prime}, a_{-i}\right)+\theta\left(a_{i}-a_{i}^{\prime}\right)-g_{i}\left(a_{i}, a_{-i}\right)+g_{i}\left(a_{i}^{\prime}, a_{-i}\right)-\theta^{\prime}\left(a_{i}-a_{i}^{\prime}\right) \\
& \quad=\left(\theta-\theta^{\prime}\right)\left(a_{i}-a_{i}^{\prime}\right),
\end{aligned}
$$

that is, $u$ satisfies the state monotonicity assumption A3. For all $a_{i}<m_{i}$ and sufficiently large $\bar{\theta}_{i} \geq 0$ we have

$$
u\left(m_{i}, a_{-i}, \bar{\theta}_{i}\right)-u\left(a_{i}, a_{-i}, \bar{\theta}_{i}\right)=g_{i}\left(m_{i}, a_{-i}\right)-g_{i}\left(a_{i}, a_{-i}\right)+\bar{\theta}_{i}\left(m_{i}-a_{i}\right)>0,
$$

so $m_{i}$ is the dominant action for $\theta \geq \bar{\theta}_{i}$. Analogously, there exists $\underline{\theta}_{i} \leq 0$ such that $a_{i}=0$ is the dominant action for $\theta \leq \underline{\theta}_{i}$. Choosing $\bar{\theta}=\max \left\{\bar{\theta}_{i}\right\}$ and $\underline{\theta}=\min \left\{\underline{\theta}_{i}\right\}$, $u$ satisfies A2. As we let $\theta$ be distributed over a large interval containing $[\underline{\theta}, \bar{\theta}]$ and choose $f$ arbitrarily, we have constructed a global game $G_{v}(u, \phi, f)$ in which $g$ is the embedded game $g(0)$.

FMP show that generically, the limit profile of a global game determines a unique equilibrium of an embedded game. The following lemma slightly strengthens FMP's theorem 2 for games with finite action sets. It shows, in addition, that these equilibria are generically strict.

Lemma 3. Let $G_{v}(u, \phi, f)$ be a global game and $s^{f}$ its essentially unique limit strategy profile. Then for any state parameter $\theta^{*}, \bar{s}^{f}\left(\theta^{*}\right)$ and $\underline{s}^{f}\left(\theta^{*}\right)$ are Nash equilibria of the embedded game $g\left(\theta^{*}\right)$. Moreover, if $s^{f}$ is continuous at $\theta$, then $\bar{s}^{f}\left(\theta^{*}\right)=\underline{s}^{f}\left(\theta^{*}\right)=a^{*}$ is a strict Nash equilibrium of the embedded game $g\left(\theta^{*}\right)$.

Proof. Let $\theta^{*}$ be given, and first consider the case where $s^{f}$ is continuous at $\theta^{*}$. Since the joint action space $A$ is finite, there is $\delta>0$ such that $s^{f}$ is constant on the interval $\left[\theta^{*}-\delta, \theta^{*}+\delta\right]$. Now consider the family of simplified global games $G_{v}(u, f)$ as $v$ tends to 0 . Recall that each $G_{v}(u, f)$ has an essentially unique equilibrium strategy profile $s_{v}^{f}$, and that $s_{v}^{f}$ converges towards $s^{f}$ in horizontal distance as $v$ tends to zero. Since $s^{f}$ is constant on the interval $\left[\theta^{*}-\delta, \theta^{*}+\delta\right]$, there must be some $\bar{v}>0$ such that if $v<\bar{v}$ then $s_{v}^{f}$ is equal to $s^{f}\left(\theta^{*}\right)$ on the interval $\left[\theta^{*}-\frac{\delta}{2}, \theta^{*}+\frac{\delta}{2}\right]$, and hence constant on this subinterval.

Choose some $v^{*}<\min \{\delta / 2, \bar{v}\}$. and assume that in the simplified global game $G_{v^{*}}(u, f)$ all players follow the strategy profile $s_{v^{*}}^{f}$. Now suppose that in this game some player $i$ receives the signal $x_{i}=\theta^{*}$. Then $i$ knows that all other players receive signals in the interval $\left[\theta^{*}-\frac{\delta}{2}, \theta^{*}+\frac{\delta}{2}\right]$. As $s_{v^{*}}^{f}$ is constant and equal to $s^{f}\left(\theta^{*}\right)=a^{*}$ on this interval, $i$ 's opponents play the action profile $a_{-i}^{*}$ that is determined by $s^{f}\left(\theta^{*}\right)$. As $s_{v^{*}}^{f}$ is an equilibrium strategy profile, we know that $s_{i}^{f}\left(\theta^{*}\right)=a_{i}^{*}$ must be a best reply to $a_{-i}^{*}$ for player $i$. Moreover, since player $i$ receives the signal $x_{i}=\theta^{*}$, she believes her payoff function is exactly like in $g\left(\theta^{*}\right)$. Thus $a_{i}^{*}$ is in fact a best reply to $a_{-i}^{*}$ in the complete information game $g\left(\theta^{*}\right)$. Since this is true for any player $i$ that receives the signal $\theta^{*}, a_{i}^{*}$ is a best reply to $a_{-i}^{*}$ in the game $g\left(\theta^{*}\right)$ for all $i \in I$, viz. a Nash equilibrium.

If $s^{f}$ is continuous at $\theta^{*}$ then it is constant and continuous on the interval $\left[\theta^{*}-\delta, \theta^{*}+\delta\right]$. By the above argument, for any $\theta^{\prime} \in\left(\theta^{*}-\delta, \theta^{*}+\delta\right), s^{f}\left(\theta^{\prime}\right)=s^{f}\left(\theta^{*}\right)=a^{*}$ is a Nash 
equilibrium of any game $g\left(\theta^{\prime}\right)$. Now suppose $a^{*}$ is not a strict equilibrium of $g\left(\theta^{*}\right)$, i.e. there are $i \in I$ and $a_{i} \neq a_{i}^{*} \in A_{i}$ such that $a_{i}$ is a best reply to $a_{-i}^{*}$ in the game $g\left(\theta^{*}\right)$. Then, by the state monotonicity assumption A3, for either $\theta^{\prime}<\theta^{*}$ or $\theta^{\prime}>\theta^{*}$, $u\left(a_{i}, a_{-i}^{*}, \theta^{\prime}\right)>u\left(a_{i}^{*}, a_{-i}^{*}, \theta^{\prime}\right)$. Yet this contradicts that $a^{*}$ is a Nash equilibrium for all games $g\left(\theta^{\prime}\right), \theta^{\prime} \in\left(\theta^{*}-\delta, \theta^{*}+\delta\right)$. Hence, $a^{*}$ must be a strict equilibrium of $g\left(\theta^{*}\right)$ after all.

If $s^{f}$ is discontinuous at $\theta^{*}$, we know it is at least constant and continuous on the open interval $\left(\theta^{*}, \theta^{*}+\delta^{\prime}\right)$ for some $\delta^{\prime}>0$. By the above argument, for any strictly positive $\varepsilon<\delta^{\prime}$, $s^{f}\left(\theta^{*}+\varepsilon\right)=a^{*}$ is a Nash equilibrium of the game $g\left(\theta^{*}+\varepsilon\right)$. Hence for all $i \in I, a_{i} \in A_{i}$, and all $\varepsilon$ satisfying $0<\varepsilon<\delta^{\prime}$ we have:

$$
u_{i}\left(a_{i}^{*}, a_{-i}^{*}, \theta^{*}+\varepsilon\right) \geq u_{i}\left(a_{i}, a_{-i}^{*}, \theta^{*}+\varepsilon\right) .
$$

The continuity of $u$ in $\theta$ (assumption A4) implies that for all $i \in I, a_{i} \in A_{i}$,

$$
\lim _{\varepsilon \searrow 0} u_{i}\left(a_{i}^{*}, a_{-i}^{*}, \theta^{*}+\varepsilon\right)=u_{i}\left(a_{i}^{*}, a_{-i}^{*}, \theta^{*}\right) \geq u_{i}\left(a_{i}, a_{-i}^{*}, \theta^{*}\right)=\lim _{\varepsilon \searrow 0} u_{i}\left(a_{i}, a_{-i}^{*}, \theta^{*}+\varepsilon\right) .
$$

So $a^{*}=\lim _{\varepsilon \searrow 0} s^{f}\left(\theta^{*}+\varepsilon\right)=\bar{s}^{f}\left(\theta^{*}\right)$ is a Nash equilibrium of $g\left(\theta^{*}\right)$. By a dual argument, $\underline{s}^{f}(\theta)$ is also a Nash equilibrium of $g\left(\theta^{*}\right)$.

Combined, lemmas 2 and 3 show that, at least technically, global games can be viewed as equilibrium refinement for all games of strategic complementarities. By embedding such a game $g$ into a global game $G_{v}(u, \phi, f)$, and taking $v \rightarrow 0$, we find two distinguished equilibria of $g=g\left(\theta^{*}\right)$, namely $\underline{s}^{f}\left(\theta^{*}\right)$ and $\bar{s}^{f}\left(\theta^{*}\right) \in A$, which generically coincide. FMP proved that this equilibrium selection is independent of the prior distribution $\phi$, but may depend on the noise structure $f$. In the following subsection, we will show that it is also independent of the choice of payoff functions $u(\cdot, \theta)$ of the global game that embeds $g$.

\section{§3.2. Attainability}

Let $g$ be a complete information game of strategic complementarities. The following incomplete information game, constructed around $g$, will be central to the rest of our results.

Definition. A lower-f-elaboration, $\underline{e}(g, f)$, of $g$, is defined as the following incomplete information game. The state parameter $\theta$ is uniformly distributed over an interval $\left[-\frac{1}{2}, R\right]$, with $R \geq R^{*}:=\sum_{i \in I}\left(m_{i}+1\right)$. All individuals receive a noisy signal $x_{i}=\theta+\eta_{i}$ about the true state, with each $\eta_{i}$ drawn according to the density $f_{i}$, the support of which is a subset of $\left[-\frac{1}{2}, \frac{1}{2}\right]$. The random variables $\left\{\theta, \eta_{1}, \ldots, \eta_{I}\right\}$ are independently distributed. Players' payoffs $u_{i}$ are given by

$$
u_{i}\left(a_{i}, a_{-i}, x_{i}\right)= \begin{cases}\tilde{u}_{i}\left(a_{i}, a_{-i}\right) & \text { if } x_{i}<0 \\ g_{i}\left(a_{i}, a_{-i}\right) & \text { if } x_{i} \geq 0\end{cases}
$$

with $\tilde{u}_{i}$ being an arbitrary payoff function that makes the least action dominant, e.g. for all $a_{-i}, \tilde{u}_{i}\left(0, a_{-i}\right)=1$ and $\tilde{u}_{i}\left(a_{i}, a_{-i}\right)=0$ when $a_{i} \neq 0$. An upper-f-elaboration, $\bar{e}(g, f)$, is 


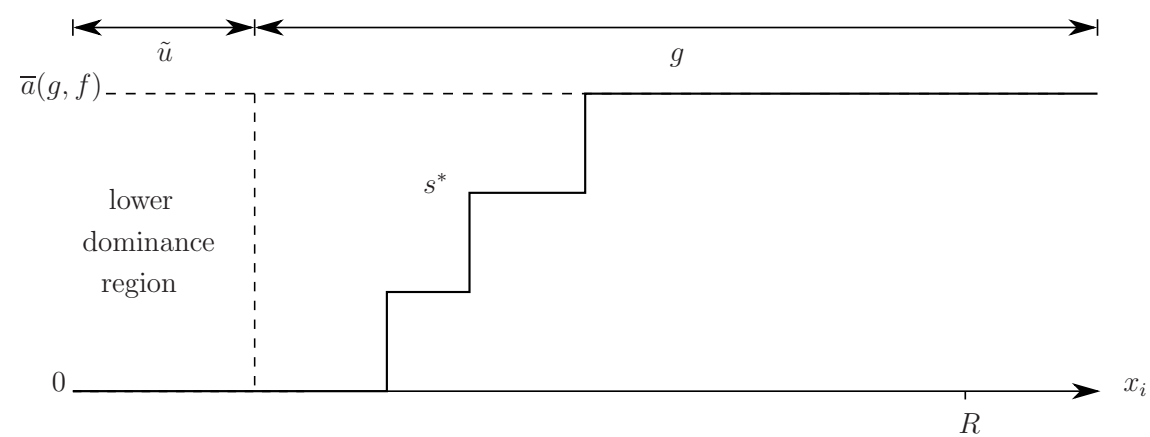

FigURE 3. A lower-f-elaboration such that the greatest equilibrium strategy profile, $s^{*}$, attains $\bar{a}(g, f)$.

defined dually: $\theta$ is distributed over $\left[L, \frac{1}{2}\right]$, where $L \leq L^{*}:=-R^{*}$, and for each player $i$ the greatest action is dominant for all signals $x_{i}>0$.

Just as for global games, we define strategy profiles, expected payoffs $u_{i}\left(a_{i} \mid s, x_{i}\right)$, upperbest reply strategy profiles $\hat{\beta}(s)$, and equilibrium strategy profiles in $\underline{e}(g, f)$. In any equilibrium strategy profile $s$ of $\underline{e}(g, f)$ we must have $s(x)=0$ for signals $x<0$. In equilibrium, the behaviour of players receiving signals smaller than 0 affects the choices of players receiving signals greater than 0 by the usual "infection" argument (cf. Morris et al. [16]). As a consequence, even if the action profile $a \in A$ is a Nash equilibrium of the complete information game $g$, the action profile $a$ is not necessarily played in any equilibrium strategy profile $s$ of $\underline{e}(g, f)$. We say an equilibrium strategy profile $s$ of the lower- $f$-elaboration $\underline{e}(g, f)$ attains $a$ if $s(x) \geq a$ for some $x \in\left[-\frac{1}{2}, R\right]$ (and, dually, an equilibrium strategy profile $s$ of an upper- $f$-elaboration $\bar{e}(g, f)$ attains $a$ if $s(x) \leq a$ for some $\left.x \in\left[L, \frac{1}{2}\right]\right)$.

We can use standard results (e.g. Vives [28]) on games with strategic complementarities to analyse lower- $f$-elaborations. The upper-best reply function $\hat{\beta}(s)$ is monotonic, and hence there is a greatest equilibrium strategy profile $s^{*}$, which is increasing. The greatest action profile that $s^{*}$ attains is $s^{*}\left(R+\frac{1}{2}\right)$.

Definition. An action profile $a \in A$ is attained from below under $f$ if in some lower$f$-elaboration of $g$, the greatest equilibrium strategy profile attains $a$ (see figure 3 ). In particular, we denote the greatest action profile that is attained from below under $f$ by $\bar{a}(g, f)$. We define attained from above under $f$ dually, and in particular we define $\underline{a}(g, f)$ as the least action profile used in the least equilibrium strategy profile of some upper- $f$-elaboration.

In the remainder of this section we will prove that in order to determine the global game selection in $g$, it suffices to look at $\bar{a}(g, f)$ and $\underline{a}(g, f)$. A first easy but useful observation is that to determine $\bar{a}(g, f)$ it actually suffices to look at any one lower- $f$-elaboration. (Of course, a dual observation holds for $\underline{a}(g, f))$.

Lemma 4. Let $\underline{e}(g, f)$ be any lower-f-elaboration, and $s^{*}$ its greatest equilibrium strategy profile. Then $s^{*}\left(R^{*}\right)=\bar{a}(g, f)$. 
Proof. Consider any lower- $f$-elaboration $\underline{e}^{\prime}(g, f)$ of $g$ with a greatest equilibrium strategy profile $s^{* *}$ that attains $\bar{a}(g, f)$. Since $s^{* *}$ is increasing and the joint action space $A$ is finite, we can identify $s^{* *}$ with a finite sequence $z_{1}, z_{2}, \ldots, z_{k}$, with $k \leq R^{*}$, of jump points, at which players switch to greater action profiles. If players follow the strategy profile $s^{* *}$, a small change in the jump point $z_{n}$ would influence their expected payoffs compared with $s^{* *}$ only at signals in the interval $\left[z_{n-1}, z_{n+1}\right]$. Thus the maximum distance between any two adjacent jump points $z_{n}$ and $z_{n+1}$ must be less then 1 . Otherwise, if $s^{* *}$ is an equilibrium strategy profile then, for sufficiently small $\varepsilon$, the similarly increasing strategy profile determined by the jump points

$$
z_{1}, z_{2}, \ldots, z_{n-1}, z_{n}-\varepsilon, \ldots, z_{k}-\varepsilon
$$

would be an equilibrium strategy profile as well, contradicting the maximality of $s^{* *}$. But if the distance between any two adjacent jump points is less then 1 , then $z_{k} \leq R^{*}$. Or, equivalently, $s^{* *}$ attains $a \in A$ if and only if $a \leq s^{* *}\left(R^{*}\right)=\bar{a}(g, f)$.

Now we may verify that the strategy profile given by the jump points $z_{1}, z_{2}, \ldots, z_{k}$ can also be interpreted as the greatest equilibrium strategy profile $s^{*}$ of $\underline{e}(g, f)$.

Our first main result is an immediate consequence of our characterisation of the global game selection process in terms of $\bar{a}(g, f)$ and $\underline{a}(g, f)$.

Theorem 5. Let $G^{v}(u, \phi, f)$ be any global game. The global game selection at any state parameter $\theta$ depends solely on the noise structure $f$ and on the complete information game $g(\theta)$, and is independent of $u$ and $\phi$.

More precisely, if $s^{f}$ is the essentially unique limit strategy profile of $G^{v}(u, \phi, f)$, and $g=g\left(\theta^{*}\right)$ then $\bar{s}^{f}\left(\theta^{*}\right)=\bar{a}(g, f)$ and $\underline{s}^{f}\left(\theta^{*}\right)=\underline{a}(g, f)$.

The irrelevance of the prior distribution $\phi$ for the global game selection was already shown by FMP. It may be surprising that the choice of payoff functions surrounding $g(\theta)$ is irrelevant as well. After all, the global game selection process is often described as an infection process, starting from the high and low parameter regions. Thus, one might think that choosing to embed $g$ at $\theta$ close to the lower dominance threshold $\underline{\theta}$ might influence the global game selection so that it selects a lower equilibrium compared to an embedding of $g$ close to $\bar{\theta}$. However, theorem 5 tells us that will not be the case.

Another way to think about theorem 5 is the following. In economic applications, the state parameter $\theta$ is typically interpreted as an economic fundamental affecting the decision problem of players. But there may be several economic variables that are candidates for the parameter $\theta$. Theorem 5 tells us that the choice of the fundamental used to perturb the decision problem is irrelevant: the global game selection will be the same. It is determined by the payoff structure of the unperturbed game.

The theorem is an immediate consequence of the following three lemmas. The first lemma is inspired by a construction in the proof of theorem 4 in FMP. 
Lemma 6. Let $G_{v}(u, \phi, f)$ be any global game that embeds $g$ and $s^{f}$ its essentially unique limit strategy profile. Let $g=g\left(\theta^{*}\right)$. We have:

$$
\bar{s}^{f}\left(\theta^{*}\right) \geq \bar{a}(g, f) \text {, and dually, } \underline{s}^{f}\left(\theta^{*}\right) \leq \underline{a}(g, f) .
$$

Proof. We prove the first inequality. Without loss of generality assume that $\theta^{*}=0$.

For the moment, fix some $v^{*}$ such that $0<v^{*} \leq 1$. Consider the simplified global game $G_{v^{*}}(u, f)$. We will want to assume that its random state parameter $\theta$ is distributed over an interval $[L, R]$ where $L \leq \min \left\{\underline{\theta}-1,-\frac{1}{2}\right\}$ and $R \geq \max \left\{\bar{\theta}+1, R^{*}\right\}$. This will allow us to compare the simplified global game with a lower- $f$-elaboration of $g$ later in the proof. For $G_{v^{*}}(u, f)$, this might mean extending the range over which $\theta$ is distributed further into the dominance regions. But that will not change its essentially unique equilibrium profile $s_{v^{*}}^{f}$, other than enlarging the range in which dominant actions are prescribed. So this is without loss of generality.

Consider the lower- $f$-elaboration $\underline{e}(g, f)$, with $\theta$ distributed on $\left[\frac{1}{2}, R^{*}\right]$. Its greatest equilibrium strategy profile $s^{*}$ is increasing and satisfies $s^{*}(x)=\bar{a}(g, f)$ for $x \geq R^{*}$. We define the profile $s_{v^{*}}$ as follows:

$$
\text { for all } \mathrm{i} \in I, \quad s_{v^{*}, i}(x):= \begin{cases}0 & \text { if } L \leq x<-\frac{1}{2} v^{*}, \\ s_{i}^{*}\left(x / v^{*}\right) & \text { if }-\frac{1}{2} v^{*} \leq x \leq v^{*} R^{*}, \\ \bar{a}(g, f) & \text { if } v^{*} R^{*}<x \leq R .\end{cases}
$$

Next, we will compare the simplified global game with a "compressed" version of $\underline{e}(g, f)$, where all individual noise variables $\eta_{i}$ have been scaled by the factor $v^{*}$, and $\theta$ is distributed uniformly on $\left[-\frac{1}{2} v^{*}, v^{*} R^{*}\right]$. We denote this compressed lower- $f$-elaboration of $g$ by $\underline{e}_{v^{*}}(g, f)$. Compressing the elaboration amounts merely to a relabeling of signals. Therefore, the restriction of $s_{v^{*}}$ to the signal space of $\underline{e}_{v^{*}}(g, f)$ is an equilibrium strategy profile of $\underline{e}_{v^{*}}(g, f)$. Note also that in the games $G_{v^{*}}(u, f)$ and $\underline{e}_{v^{*}}(g, f)$ the distributions of opponents' signals conditional on a player's own signal are identical, for all signals in the interval $\left[-\frac{1}{2} v^{*}, v^{*} R^{*}\right]$.

Now suppose that players follow the strategy profile $s_{v^{*}}$ in the simplified global game $G_{v^{*}}(u, f)$. For any player $i$, and any signal $x_{i}<0,0$ is a dominant action in $\underline{e}_{v^{*}}(g, f)$, so that $s_{v^{*}, i}\left(x_{i}\right)=0 \leq \hat{\beta}\left(s_{v^{*}}\right)_{i}\left(x_{i}\right)$ in $G_{v^{*}}(u, f)$.

For $x_{i}=0$, we have $s_{v^{*}, i}\left(x_{i}\right) \leq \hat{\beta}\left(s_{v^{*}}\right)_{i}\left(x_{i}\right)$, since $s_{v^{*}}$ is an equilibrium of $\underline{e}_{v^{*}}(g, f)$, and therefore $s_{v^{*}, i}(0)$ is a best reply to $s_{v^{*}, i}$ under the payoff function $u(\cdot, 0)=g$. In particular, this means $s_{v^{*}, i}\left(x_{i}\right)$ must be weakly smaller than the greatest best reply under the payoff function $u(\cdot, 0)$.

For $x_{i}>0, s_{v^{*}, i}\left(x_{i}\right) \leq \hat{\beta}\left(s_{v^{*}}\right)_{i}\left(x_{i}\right)$, since $s_{v^{*}, i}\left(x_{i}\right)$ is the best reply to $s_{v^{*}, i}$ under the payoff function $u(\cdot, 0)=g$, and hence the greatest best reply under the payoff function $u\left(\cdot, x_{i}\right)$ must be weakly greater than $s_{v^{*}, i}\left(x_{i}\right)$ by assumption A3.

In sum, $s_{v^{*}} \leq \hat{\beta}\left(s_{v^{*}}\right)$, and therefore an upper-best reply iteration starting at $s_{v^{*}}$ yields a monotonically increasing sequence of strategy profiles that converges to the essentially unique, increasing equilibrium strategy profile $s_{v^{*}}^{f}$ of $G_{v^{*}}(u, f)$. This shows $s_{v^{*}}^{f} \geq s_{v^{*}}$. For 
the right continuous version of $s_{v^{*}}^{f}$ we find that

$$
\bar{s}_{v^{*}}^{f}\left(v^{*} R\right) \geq s_{v^{*}}\left(v^{*} R^{*}\right)=s^{*}\left(R^{*}\right)=\bar{a}(g, f) .
$$

Since $\bar{s}_{v^{*}}^{f}$ is increasing, it follows that for all $x \geq v^{*} R^{*}, \bar{s}_{v^{*}}^{f}(x) \geq \bar{a}(g, f)$.

Since the choice of $v^{*}$ was arbitrary, the above argument shows that for all $\varepsilon>0$, there is $\bar{v}>0$ such that $\bar{s}_{v}^{f}(\varepsilon) \geq \bar{s}_{v}^{f}\left(v R^{*}\right) \geq \bar{a}(g, f)$ for all $v \leq \bar{v}$ (just take $\left.\bar{v}=\varepsilon / R^{*}\right)$. Hence:

$$
\text { for all } \varepsilon>0, \quad \lim _{v \searrow 0} \bar{s}_{v}^{f}(\varepsilon) \geq \bar{a}(g, f)
$$

implying

$$
\bar{s}^{f}(0)=\lim _{\varepsilon \searrow 0}\left(\lim _{v \searrow 0} \bar{s}_{v}^{f}(\varepsilon)\right) \geq \bar{a}(g, f)
$$

as claimed.

The next two lemmas establish that the converse of lemma 6 also holds.

Lemma 7. Let $G^{v}(u, \phi, f)$ be any global game that embeds $g$ and $s^{f}$ its essentially unique limit strategy profile. Let $g=g\left(\theta^{*}\right)$, and assume that $s^{f}$ is continuous at $\theta^{*}$. Then we have:

$$
\bar{s}^{f}\left(\theta^{*}\right) \leq \bar{a}(g, f), \text { and dually, } \underline{s}^{f}\left(\theta^{*}\right) \geq \underline{a}(g, f) .
$$

Proof. We will prove the first inequality. Consider again the simplified global game $G_{v}(u, f)$. By (A2) there is some $\underline{\theta}$ such that for signals smaller than $\underline{\theta}$, the action 0 is dominant for all players. Without loss of generality we will assume that $\underline{\theta}=0$. Since the joint action space is finite, continuity at the point $\theta^{*}$ implies that, for some $\delta>0, \bar{s}^{f}$ is constant, and equal to some $a^{*} \in A$, on the interval $\left[\theta^{*}-\delta, \theta^{*}+\delta\right]$. Since $\bar{s}_{v}^{f}$, the right continuous equilibrium strategy profile of the simplified global game $G_{v}(u, f)$, converges towards $\bar{s}^{f}$ in horizontal distance, there must be $\bar{v}>0$ such that for $v<\bar{v}, s^{f}$ equals $\bar{s}_{v}^{f}$ on the subinterval $\left[\theta^{*}-\delta / 2, \theta^{*}+\delta / 2\right]$.

Fix some $v^{*}<\min \{\delta / 2, \bar{v}\}$. Consider the "compressed" lower- $f$-elaboration $\underline{e}_{v^{*}}(g, f)$, where all individual noise variables $\eta_{i}$ have been scaled by the factor $v^{*}$, and $\theta$ is distributed uniformly on the interval $\left[-\frac{1}{2}, R\right]$, with $R$ the same as in the simplified global game. Assume that in this game players use the strategy profile $s$ given by:

$$
\text { for all } i \in I, \quad s_{i}(x)= \begin{cases}\bar{s}_{v^{*}, i}^{f}\left(x_{i}\right) & \text { if } x_{i} \leq \theta^{*}, \\ \bar{s}_{v^{*}, i}^{f}\left(\theta^{*}\right) & \text { if } x_{i}>\theta^{*}\end{cases}
$$

For any player $i$, and any signal $x_{i}<0,0$ is a dominant action for $i$ both in $\underline{e}_{v^{*}}(g, f)$ and in $G_{v^{*}}(u, f)$. So in the game $\underline{e}_{v^{*}}(g, f)$, we have

$$
\text { for } x_{i}<0, \quad \hat{\beta}(s)_{i}\left(x_{i}\right)=0=\bar{s}_{v^{*}, i}^{f}\left(x_{i}\right)=s_{i}\left(x_{i}\right) .
$$

For $x_{i} \in\left[0, \theta^{*}\right]$, player $i$ 's opponents receive signals smaller than $\theta^{*}+\frac{\delta}{2}$, since $v^{*}<\frac{\delta}{2}$. So $i$ 's opponents behave as if they were following $\bar{s}_{v^{*}, i}^{f}\left(x_{i}\right)$. Since the distributions of the state parameter and of the signals are identical in $G_{v^{*}}(u, f)$ and $\underline{e}_{v^{*}}(g, f)$, but $i$ 's payoff function is given by $u_{i}\left(\cdot, \theta^{*}\right)$ in $\underline{e}_{v^{*}}(g, f)$ and by $u_{i}\left(\cdot, x_{i}\right)$ in $G_{v^{*}}(u, f)$, in the game $\underline{e}_{v^{*}}(g, f)$ 
we have

$$
\text { for } 0 \leq x_{i} \leq \theta^{*}, \quad \hat{\beta}(s)_{i}\left(x_{i}\right)=\hat{\beta}\left(\bar{s}_{v^{*}}^{f}\right)_{i}\left(x_{i}\right) \geq \bar{s}_{v^{*}, i}^{f}\left(x_{i}\right)=s_{i}\left(x_{i}\right),
$$

where the inequality follows from the state monotonicity assumption A3 and the fact that $\bar{s}_{v^{*}}^{f}$ is the greatest equilibrium profile of $G_{v^{*}}(u, f)$.

For $x_{i}>\theta^{*}$, player $i$ 's opponents receive signals greater than $\theta^{*}-\frac{\delta}{2}$. Since for such signals $s$ is constant and equal to $\bar{s}_{v^{*}}^{f}\left(\theta^{*}\right)=a^{*}$, player $i$ 's opponents will use the action profile $a_{-i}^{*}$. By lemma 3, $a^{*}$ is a Nash equilibrium under the payoff functions of the game $g$. This means that in $\underline{e}_{v^{*}}(g, f)$ we have

$$
\text { for } x_{i}>\theta^{*}, \quad \hat{\beta}(s)_{i}\left(x_{i}\right) \geq a_{i}=\bar{s}_{v^{*}, i}^{f}\left(x_{i}\right)=s_{i}\left(x_{i}\right) .
$$

In sum, $\hat{\beta}(s) \geq s$. Therefore an upper-best reply iteration in the game $\underline{e}_{v^{*}}(g, f)$ starting from $s$ yields a monotonically increasing sequence of strategy profiles that converge to an equilibrium profile $s^{*} \geq s$. It follows that $s^{*}\left(\theta^{*}\right) \geq s\left(\theta^{*}\right)=\bar{s}^{f}\left(\theta^{*}\right)$. Since compressing an elaboration amounts to a relabelling of signals, in the equivalent uncompressed lower$f$-elaboration there is an equilibrium strategy profile $s^{* *}$ such that $s^{* *}\left(\theta^{*} / v^{*}\right) \geq \bar{s}^{f}\left(\theta^{*}\right)$, Thus $\bar{s}^{f}\left(\theta^{*}\right)$ is attained from below under $f$, implying the inequality $\bar{s}^{f}\left(\theta^{*}\right) \leq \bar{a}(g, f)$.

Lemma 8. Let $G^{v}(u, \phi, f)$ be any global game that embeds $g$ and $s^{f}$ its essentially unique limit strategy profile. Let $g=g\left(\theta^{*}\right)$, and assume that $s^{f}$ is not continuous at $\theta^{*}$. Then we have:

$$
\bar{s}^{f}\left(\theta^{*}\right) \leq \bar{a}(g, f) \text {, and dually, } \underline{s}^{f}\left(\theta^{*}\right) \geq \underline{a}(g, f) .
$$

Proof. We will again prove the first inequality. Let $\left\{\theta_{n}\right\}_{n \in \mathbb{N}}$ be a sequence that converges to $\theta^{*}$ from above. By lemma 7 , for any $\theta_{n}$, the greatest equilibrium profile $s^{\theta_{n}}$ of every lower- $f$-elaboration $\underline{e}\left(g\left(\theta_{n}\right), f\right)$ attains $\bar{s}^{f}\left(\theta_{n}\right)$, and hence attains $\bar{s}^{f}\left(\theta^{*}\right)$. Moreover, by the state monotonicity assumption (A3), the sequence of profiles $s^{\theta_{n}}$ converges to $s^{*}=$ $\inf \left\{s^{\theta_{n}} \mid n \in \mathbb{N}\right\}$ in monotonically decreasing fashion. Since the joint action space $A$ is finite, for each $x$ there is $n_{x}$ such that $s^{\theta_{n}}(x)=s^{*}(x)$.

Denote by $u_{i}\left(a_{i} \mid s, x_{i} ; \theta_{n}\right)$ the expected payoff of player $i$ when she plays the action $a_{i}$ against the strategy profile $s$ in the lower- $f$-elaboration $\underline{e}\left(g\left(\theta_{n}\right), f\right)$. We claim that for all $\varepsilon>0$ and all signal tuples $x \geq 0$, there is an $\bar{n}$ such that $\theta^{*}<\theta_{\bar{n}}<\theta^{*}+\varepsilon$ and such that

$$
\forall i \forall a_{i}, \quad u_{i}\left(s_{i}^{*}\left(x_{i}\right) \mid s^{*}, x_{i} ; \theta_{\bar{n}}\right) \geq u_{i}\left(a_{i} \mid s^{*}, x_{i} ; \theta_{\bar{n}}\right) .
$$

But then, from the continuity of $u$ in the state parameter (assumption A4), we deduce that for all $x_{i} \geq 0$ we must have

$$
\forall i \forall a_{i}, \quad u_{i}\left(s_{i}^{*}\left(x_{i}\right) \mid s^{*}, x_{i} ; \theta^{*}\right) \geq u_{i}\left(a_{i} \mid s^{*}, x_{i} ; \theta^{*}\right),
$$

viz. $s^{*}$ is an equilibrium strategy profile of $\underline{e}(g, f)$. Clearly $s^{*}$ attains $\bar{s}^{f}\left(\theta^{*}\right)$.

To prove the claim, fix some $x \geq 0$ and note that for all $n>n_{x}$ we have:

$$
\forall i \forall a_{i}, \quad u_{i}\left(s_{i}^{*}\left(x_{i}\right) \mid s^{\theta_{n}}, x_{i} ; \theta^{n}\right) \geq u_{i}\left(a_{i} \mid s^{\theta_{n}}, x_{i} ; \theta^{n}\right),
$$


since $s^{\theta_{n}}$ is an equilibrium profile of $\underline{e}\left(g\left(\theta_{n}\right), f\right)$. In particular we may choose $\bar{n}>n_{x}$ such that $\theta^{\bar{n}}<\theta^{*}+\varepsilon$. In that case we find, for all $n>\bar{n}$,

$$
\begin{aligned}
\forall i \text { and } \forall a_{i} \leq s_{i}^{*}\left(x_{i}\right), \quad 0 & \geq u_{i}\left(a_{i} \mid s^{\theta_{n}}, x_{i} ; \theta_{n}\right)-u_{i}\left(s_{i}^{*}\left(x_{i}\right) \mid s^{\theta_{n}}, x_{i} ; \theta_{n}\right) \\
& \geq u_{i}\left(a_{i} \mid s^{\theta_{n}}, x_{i} ; \theta_{\bar{n}}\right)-u_{i}\left(s_{i}^{*}\left(x_{i}\right) \mid s^{\theta_{n}}, x_{i} ; \theta_{\bar{n}}\right) \quad \text { by (A3), } \\
\forall i \text { and } \forall a_{i} \geq s_{i}^{*}\left(x_{i}\right), \quad 0 & \geq u_{i}\left(a_{i} \mid s^{\theta_{\bar{n}}}, x_{i} ; \theta_{\bar{n}}\right)-u_{i}\left(s_{i}^{*}\left(x_{i}\right) \mid s^{\theta_{\bar{n}}}, x_{i} ; \theta_{\bar{n}}\right) \\
& \geq u_{i}\left(a_{i} \mid s^{\theta_{n}}, x_{i} ; \theta_{\bar{n}}\right)-u_{i}\left(s_{i}^{*}\left(x_{i}\right) \mid s^{\theta_{n}}, x_{i} ; \theta_{\bar{n}}\right) \quad \text { by (A1). }
\end{aligned}
$$

So we conclude that for all $n>\bar{n}$

$$
\forall i \forall a_{i}, \quad u_{i}\left(s_{i}^{*}\left(x_{i}\right) \mid s^{\theta_{n}}, x_{i} ; \theta^{\bar{n}}\right) \geq u_{i}\left(a_{i} \mid s^{\theta_{n}}, x_{i} ; \theta^{\bar{n}}\right) .
$$

Since $\left\{s^{\theta_{n}}\right\}_{n \in \mathbb{N}}$ converges to $s^{*}$, by the monotone convergence theorem we now find

$$
\forall i \forall a_{i}, \quad u_{i}\left(s_{i}^{*}\left(x_{i}\right) \mid s^{*}, x_{i} ; \theta^{\bar{n}}\right) \geq u_{i}\left(a_{i} \mid s^{*}, x_{i} ; \theta^{\bar{n}}\right) .
$$

\section{Noise Independence}

A game $g$ embedded at state parameter $\theta^{*}$ in a global game $G_{v}(u, \phi, f)$ is called noise independent if its limit strategy profile $s^{f}$ takes on the same values at $\theta^{*}$ regardless of the choice of $f$. Theorem 5 says that noise independence is a property of the complete information game $g$ : a game of strategic complementarities is noise independent under one global game embedding if and only if it is noise independent under every other global game embedding.

In this section, we will use attainability to analyse how the global game selection may depend on the noise structure. Whether some action profile $a$ can be attained from below in a lower- $f$-elaboration is in essence a coordination problem among the players. For each player $i$, there must be thresholds $z_{i}^{0}, z_{i}^{1}, \ldots, z_{i}^{k}$ at which she is willing to switch to a greater action, given the action distribution of opponents' actions. The players' thresholds need to be mutually consistent under the noise structure $f$. This problem takes the simplest form - and may be solvable independent of $f$-if there are few players or few actions, or if some actions are very appealing for wide range of opposing action distributions.

The simplest non-trivial games with multiple equilibria are 2 player, 2 action games. For such $2 \times 2$ games it is known that the global game selection is noise independent; it selects the risk dominant equilibrium (Carlsson and Van Damme [4]). In a symmetric $2 \times 2$ game this means it selects the best replies to the conjecture that the opponent mixes over both actions with equal probability. If both the least and greatest actions are best replies to this conjecture, then the least is prescribed by the left continuous version of the limit strategy $s^{f}$ while the right continuous version prescribes the greatest.

But noise independence may fail quickly when the player set or the action sets of players are enlarged beyond size 2. An example of Carlsson [3] shows that noise independence may already fail in an (asymmetric) 3 player, binary action game. Oyama and Takahashi [22] show that symmetric 2 player $3 \times 3$ games are noise independent, but FMP present an example where noise independence fails in a symmetric 2 player $4 \times 4$ game. Below, 


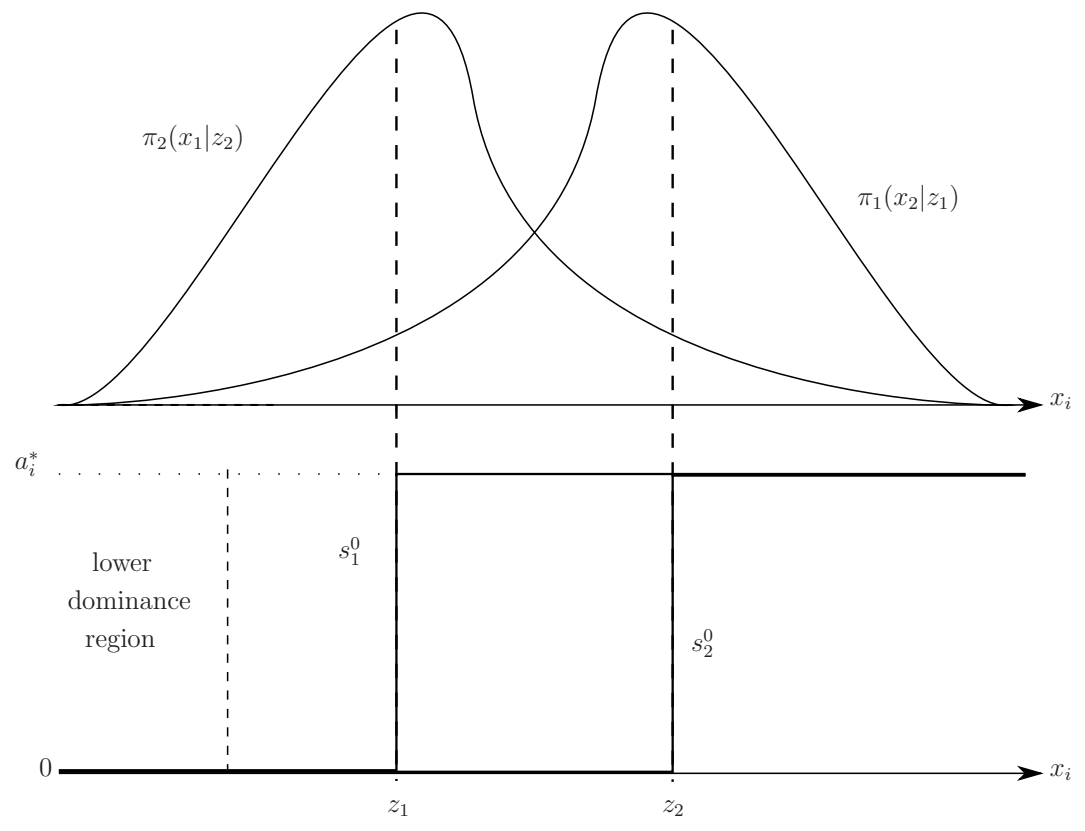

Figure 4. ToP: The thresholds $z_{i}$, at which players believe that the opponent will receive a signal $x_{-i} \geq z_{-i}$ with probability $\frac{1}{2}$.

Воттом: The starting point for the upper-best reply iteration.

we provide two further results on how many players or actions it takes to violate noise independence. We show that any 2 player game in which one player's action space is binary (i.e., every $2 \times n$ game) is noise independent. We will also give an example where it fails in an asymmetric 2 player $3 \times 3$ game. This gives a full characterisation of games where noise independence can be established simply by counting the number of players or actions.

A well known, yet restrictive, criterion that guarantees noise dependence even in many player and many action games is the " $p$-dominance" criterion.

Definition. Let $p=\left(p_{i}\right)_{i \in I}$ and $\triangle\left(A_{-i}\right)$ be the set of all probability distributions over $A_{-i}$. An action profile $a^{*}$ in $g$ is $p$-dominant if for each player $i$ and any opposing action distribution $\mu \in \triangle\left(A_{-i}\right)$ that assigns weight $\mu\left(a_{-i}^{*}\right) \geq p_{i}$ we find that

$$
\forall a_{i}, \quad \sum_{a_{-i} \in A_{-i}} \mu\left(a_{-i}\right) g_{i}\left(a_{i}^{*}, a_{-i}\right) \geq \sum_{a_{-i} \in A_{-i}} \mu\left(a_{-i}\right) g_{i}\left(a_{i}, a_{-i}\right),
$$

i.e. $a_{i}^{*}$ is a best response.

If $a^{*}$ is $p$-dominant for some $p$ with $\sum_{i \in I} p_{i}<1$, then $a^{*}$ is the global game selection (see FMP). It follows that, independent of the noise structure, $a^{*}$ is the global game selection in a two player symmetric payoff game if it is a best reply on the conjecture that the opponent will play $a_{-i}^{*}$ with probability less than $\frac{1}{2}$. The concept of attainability in a lower- $f$-elaboration enables us to see why. For any $f$ we find thresholds $z_{i} \in[0,1]$ such that $\mathbb{P}\left(x_{-i}>z_{i} \mid x_{i}=z_{i}\right)=\frac{1}{2}$ (see the top panel of figure 4). Now, consider the strategy profile $s^{0}$ where each player $i$ switches from her lowest action to $a_{i}^{*}$ at $z_{i}$ (see the bottom panel of figure 4). As each player receiving the signal $x_{i}=z_{i}$ assigns a probability of 


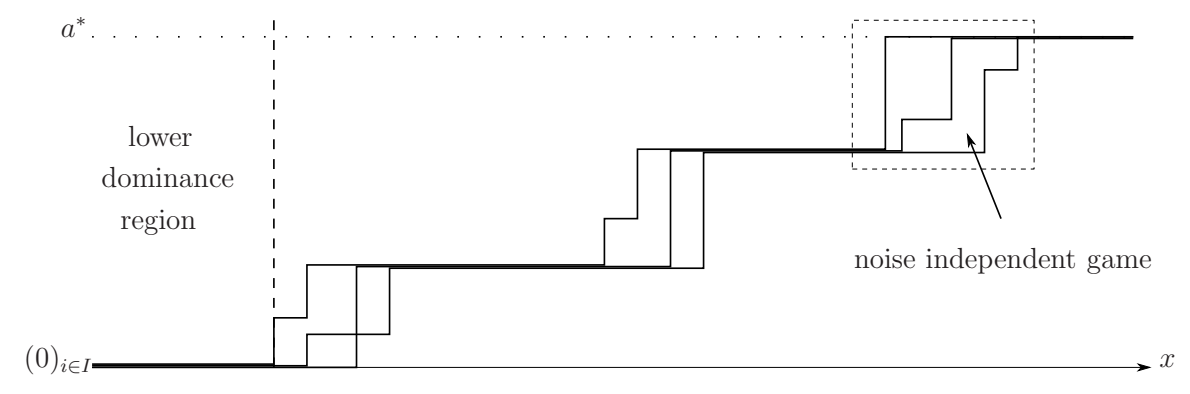

FiguRE 5. Attaining $a^{*}$ by exploiting noise independence of restricted games.

$\frac{1}{2} \geq p_{i}$ to the event that his opponent plays $a^{*}, p$-dominance guarantees that the best reply to $s^{0}$ is weakly greater than $s^{0}$. Thus, an upper-best reply iteration starting at $s^{0}$ converges towards an equilibrium strategy profile $s$ that attains $a^{*}$.

Looking at the strategy profile $s^{0}$, it becomes clear that it is unnecessarily strict to require that players are willing to switch from 0 to $a^{*}$ immediately. Allowing them to reach $a^{*}$ in many steps considerably weakens the criterion.

\section{§4.1. A Decomposition Approach to Noise Independence}

Elaborating on this idea, we will show that if there exists a suitable decomposition of a game of strategic complementarities $g$ into smaller, noise independent games, this may be a sufficient condition for noise independence of $g$ itself. Figure 5 illustrates the idea. If we can show that certain action profiles are attained in certain lower- $f$-elaborations that we obtain by restricting the strategy space of $g$, we can "patch" these strategy profiles together to obtain a strategy profile for a lower- $f$-elaboration of the full game $g$. In this case, simple known criteria for games with small action spaces may prove to be extremely useful to analyse games with bigger action spaces.

Definition. Consider a game of strategic complementarities $g$ with joint action set $A$. The greatest and least action profiles in $A$ are denoted by $m$ and 0 . For action profiles $a \leq a^{\prime}$, we define $\left[a, a^{\prime}\right]:=\left\{\tilde{a} \in A \mid a \leq \tilde{a} \leq a^{\prime}\right\}$. The restricted game $g \uparrow_{\left[a, a^{\prime}\right]}$ is defined by the restriction of the payoff functions of $g$ to the set $\left[a, a^{\prime}\right]$. We write $a \stackrel{g}{\rightarrow} a^{\prime}$ if and only if $a^{\prime}$ is the unique noise independent global game selection in $g \uparrow_{\left[a, a^{\prime}\right]}$, and conversely $a \stackrel{g}{\leftarrow} a^{\prime}$ if and only if $a$ is the unique noise independent global game selection in $g \uparrow_{\left[a, a^{\prime}\right]}$.

We write $0 \stackrel{g}{\longmapsto} a^{*}$ if there exists an increasing sequence $0<a^{1}<\cdots<a^{k}<a^{*}$ in $A$ such that $0 \stackrel{g}{\rightarrow} a^{1} \stackrel{g}{\rightarrow} \ldots \stackrel{g}{\rightarrow} a^{k} \stackrel{g}{\rightarrow} a^{*}$ and $a^{*} \stackrel{g}{\longleftarrow} m$ if there exists an increasing sequence $a^{*}<a^{k+1}<\cdots<m$ in $A$ such that $a^{*} \stackrel{g}{\leftarrow} a^{k+1} \stackrel{g}{\leftarrow} \ldots \stackrel{g}{\leftarrow} m$.

Now we may prove the following sufficient condition for noise independence.

Theorem 9. If $0 \stackrel{g}{\longmapsto} a^{*} \stackrel{g}{\longleftarrow} m$, then $a^{*}$ is the unique noise independent global game selection in $g$. More precisely, let $G_{v}(u, \phi, f)$ be any global game that embeds $g$, and $s^{f}$ its essentially unique limit strategy profile. Let $g=g\left(\theta^{*}\right)$. Then:

$$
\underline{s}^{f}\left(\theta^{*}\right)=a^{*}=\bar{s}^{f}\left(\theta^{*}\right) .
$$


Theorem 9 reveals a first connection with the literature on robustness to incomplete information. Proposition 2.7 and 3.8 in Oyama and Tercieux [23] together imply that if game with strategic complementarities can be decomposed - as above -into restriced games, each of which has a strict $p$-dominant equilibrium with sufficiently small $p$ (rather than "just" a unique global game selection), then $a^{*}$ is the unique equilibrium of $g$ that is "robust to incomplete information". The formal link to our theorem runs via the observation that if an equilibrium is robust to incomplete information, then it is also the unique global game selection (at least generically; cf. our theorem 11 and corollary 12 below), so that the conclusion of theorem 9 follows.

However, theorem 9 allows application of wide range of known criteria for noise independence besides $p$-dominance, such as the fact that all symmetric $3 \times 3$ games, all symmetric $n$-player binary games, and (as we show shortly) all $2 \times n$ games are noise independent, or indeed the robustness to incomplete information of some equilibrium of the restricted game. None of these are equivalent to the $p$-dominance criterion - the conditions under which our theorem may be applied are strictly more general. Also, its conclusion does not hinge on the fact that $a^{*}$ is a robust equilibrium. Thus theorem 9 establishes a more direct and more elementary result about noise independent global game selection.

Proof. In view of theorem 5, it suffices to prove that for any noise structure $f, \bar{a}(g, f)=$ $a^{*}=\underline{a}(g, f)$. We will prove the first equality, the second follows by duality.

Fix some arbitrary noise structure $f$. By definition, there exists an increasing sequence $0<a^{1}<a^{2}<\cdots<a^{k}<a^{*}$, such that for each adjacent pair $a, a^{\prime}$ in the sequence, the unique noise independent selection in the restricted game $g\left\lceil_{\left[a, a^{\prime}\right]}\right.$ is $a^{\prime}$. Consequently, for each adjacent pair $a, a^{\prime}$ there is a corresponding lower- $f$-elaboration $\underline{e}\left(g \uparrow_{\left[a, a^{\prime}\right]}, f\right)$ with a corresponding equilibrium strategy profile $s$ that attains $a^{\prime}$.

We claim that if $s^{1}$ attains $a^{1}$ in $\underline{e}\left(g \uparrow_{\left[0, a^{1}\right]}, f\right)$ and $s^{2}$ attains $a^{2}$ in $\underline{e}\left(g \uparrow_{\left[a^{1}, a^{2}\right]}, f\right)$, then there is a lower- $f$-elaboration $\underline{e}\left(g \uparrow_{\left[0, a^{2}\right]}, f\right)$ of $g \uparrow_{\left[0, a^{2}\right]}$ with a corresponding equilibrium strategy profile $s^{*}$ such that $s^{*}$ attains $a^{2}$. Consequently, there is a strictly shorter increasing sequence $0<a^{2}<\cdots<a^{k}<a^{*}$ with the property that for each adjacent pair $a, a^{\prime}$ in the sequence there is a corresponding lower elaboration $\underline{e}\left(g \uparrow_{\left[a, a^{\prime}\right]}, f\right)$ and a corresponding equilibrium strategy profile $s$ that attains $a^{\prime}$. By induction, it follows that $a^{*}$ is attained from below under $f$ in $g\left\lceil_{\left[0, a^{*}\right]}\right.$.

To prove the claim, assume that in $\underline{e}\left(g \uparrow_{\left[a^{0}, a^{1}\right]}, f\right), \theta$ is distributed on the interval $\left[-\frac{1}{2}, R^{1}\right]$ and that in $\underline{e}\left(g\left\lceil_{\left[a^{1}, a^{2}\right]}, f\right) \theta\right.$ is distributed on $\left[-\frac{1}{2}, R^{2}\right]$. Now consider the lower- $f$-elaboration $\underline{e}\left(g \uparrow_{\left[0, a^{2}\right]}, f\right)$ such that $\theta$ takes values in the interval $\left[-\frac{1}{2}, R^{1}+1+R^{2}\right]$, and consider the strategy profile defined by:

$$
s_{i}\left(x_{i}\right)= \begin{cases}s_{i}^{1}\left(x_{i}\right) & \text { if } x \leq R^{1}, \\ a_{i}^{1} & \text { if } R^{1}<x<R^{1}+1, \\ s_{i}^{2}\left(x_{i}-\left(R^{1}+1\right)\right) & \text { if } R^{1}+1 \leq x\end{cases}
$$


For $x_{i} \leq R^{1}$, the opposing action distribution in $\underline{e}\left(g \uparrow_{\left[a^{0}, a^{2}\right]}, f\right)$ conditional on $x_{i}$ is just like that in $\underline{e}\left(g \uparrow_{\left[0, a^{1}\right]}, f\right)$. Since $s^{1}$ is an equilibrium strategy profile of $\underline{e}\left(g \uparrow_{\left[0, a^{1}\right]}, f\right)$, we know that $s_{i}^{1}\left(x_{i}\right)$ is the best reply to $s_{-i}^{1}$ among the actions $\left\{a_{i} \in A_{i} \mid a_{i} \leq a_{i}^{1}\right\}$. So it must be that $\hat{\beta}(s)_{i}\left(x_{i}\right) \geq s_{i}^{1}\left(x_{i}\right)=s_{i}\left(x_{i}\right)$ in the game $\underline{e}\left(g\left\lceil_{\left[a^{0}, a^{2}\right]}, f\right)\right.$.

For $x_{i} \in\left[R^{1}, R^{1}+1\right]$, the opposing action distribution in $\underline{e}\left(g \uparrow_{\left[a^{0}, a^{2}\right]}, f\right)$ conditional on the signal $x_{i}$ (weakly) dominates the opposing action distribution conditional on the signal $R^{1}$, since $s$ is increasing. By strategic complementarities, $\hat{\beta}(s)_{i}\left(x_{i}\right) \geq \hat{\beta}(s)_{i}\left(R^{1}\right)=a_{i}^{1}=s_{i}\left(x_{i}\right)$.

For $x_{i} \geq R^{1}+1$, the opposing action distribution in $\underline{e}\left(g \uparrow_{\left[0, a^{2}\right]}, f\right)$ conditional on the signal $x_{i}$ is just like in that $\underline{e}\left(g \uparrow_{\left[a^{1}, a^{2}\right]}, f\right)$ conditional on the signal $x_{i}-\left(R^{1}+1\right)$. Moreover, the opposing action distribution given the signal $x_{i}$ (weakly) dominates the opposing action distribution given the signal $R^{1}$, since $s$ is increasing. This implies $\hat{\beta}(s)_{i}\left(x_{i}\right) \geq a_{i}^{1}$. Furthermore, we know that $s_{i}^{2}\left(x_{i}\right)$ is the best reply to $s_{i}^{2}$ among the actions $\left\{a_{i} \in A_{i} \mid a_{i}^{1} \leq\right.$ $\left.a_{i} \leq a_{i}^{2}\right\}$, since $s^{2}$ is an equilibrium strategy profile of $\underline{e}\left(g \uparrow_{\left[a^{1}, a^{2}\right]}, f\right)$. Combining, we must have $\hat{\beta}(s)_{i}\left(x_{i}\right) \geq s_{i}^{2}\left(x_{i}-\left(R^{1}+1\right)\right)=s_{i}\left(x_{i}\right)$ in the game $\underline{e}\left(g\left\lceil_{\left[a^{0}, a^{2}\right]}, f\right)\right.$.

In sum, $\hat{\beta}(s) \geq s$. Hence an upper-best reply iteration converges monotonically to an equilibrium strategy profile $s^{*} \geq s$. Since, by construction, $s\left(R^{1}+1+R^{2}\right)=a^{2}$, certainly $s^{*}$ attains $a^{2}$. This proves the claim.

Conclude there is a lower-f-elaboration $\underline{e}\left(g \uparrow_{\left[0, a^{*}\right]}, f\right)$ with an equilibrium strategy profile $s$ that attains $a^{*}$. Since $s$ is an equilibrium strategy profile, enlarging the joint action set from $\left[0, a^{*}\right]$ to $[0, m]$ cannot make players want to switch to smaller actions when they follow the strategy profile $s$. Hence $a^{*}$ is attained from below under $f$ given the original game $g$, implying $\bar{a}(g, f) \geq a^{*}$.

It remains to be shown that $a^{*}$ is the greatest action profile that is attained from below under $f$. Towards a contradiction, suppose there is a lower- $f$-elaboration $\underline{e}(g, f)$ of $g$ with a greatest equilibrium strategy profile $s^{*}$ that attains some $a^{* *}>a^{*}$. Let $\theta$ be distributed on $\left[-\frac{1}{2}, R\right]$ in $\underline{e}(g, f)$ and, without loss of generality, let $a^{* *}$ be the greatest action profile that $s^{*}$ attains. Recall that $s^{*}$ is increasing.

By assumption, there is a restricted game $g \uparrow_{\left[a, a^{\prime}\right]}$ with $a \stackrel{g}{\leftarrow} a^{\prime}$ and such that $a<a^{* *} \leq a^{\prime}$. Consider the lower- $f$-elaboration of $g\left\lceil_{\left[a, a^{\prime}\right]}\right.$ with $\theta$ be distributed on $\left[-\frac{1}{2}, R\right]$, and consider the strategy profile given by:

$$
\text { for all } i \in I, \quad s_{i}\left(x_{i}\right)= \begin{cases}a_{i} & \text { if } s_{i}^{*}\left(x_{i}\right)<a_{i}, \\ s_{i}^{*}\left(x_{i}\right) & \text { if } s_{i}^{*}\left(x_{i}\right) \geq a_{i} .\end{cases}
$$

For all signals $x_{i}<0, s_{i}\left(x_{i}\right)=a_{i}$ is the dominant action in $\underline{e}\left(g \uparrow_{\left[a, a^{\prime}\right]}, f\right)$. For all signals $x_{i} \geq 0$, the opposing action distribution in $\underline{e}\left(g \uparrow_{\left[a, a^{\prime}\right]}, f\right)$, conditional on $x_{i}$ and when players follow the strategy profile $s$, weakly dominates the opposing action distribution at $x_{i}$ in $\underline{e}(g, f)$ when players follow $s^{*}$. Since $s^{*}$ is an equilibrium strategy profile of $\underline{e}(g, f)$, it follows that $\hat{\beta}\left(x_{i}\right)_{i}(s) \geq s_{i}\left(x_{i}\right)$ in both lower- $f$-elaborations.

In sum, $\hat{\beta}(s) \geq s$ in $\underline{e}\left(g\left\lceil_{\left[a, a^{\prime}\right]}, f\right)\right.$. Thus an upper best-reply iteration converges to an equilibrium strategy profile $s^{* *}$ that attains $a^{* *}$. Conclude that $\bar{a}\left(g \uparrow_{\left[a, a^{\prime}\right]}, f\right) \geq a^{* *}>a$. 
Yet this contradicts that $a \stackrel{g}{\leftarrow} a^{\prime}$. So it must be that $\bar{a}(g, f)=a^{*}$ after all. As $f$ was arbitrary, this proves the theorem.

\section{§4.2. Applications}

Consider the global game game where payoffs depend on $\theta$ as in figure 6 . The $p$ dominance criterion tells us that $(c, c)$ is the unique noise independent selection for $\theta>3$, as $c$ is a best reply if one expects the opponent to chose $c$ with probability one half. If $\theta<-2,(a, a)$ is selected for the same reason, yet we cannot tell which action profile will be chosen if $\theta \in[-2,3]$ or whether the selection will be noise independent at all. However, by looking at $2 \times 2$ restricted games, and applying the risk-dominance criterion, we find that $a \stackrel{g(\theta)}{\longrightarrow} b$ and $b \stackrel{g(\theta)}{\longrightarrow} c$ for $\theta>0$ so $(c, c)$ is the unique noise independent selection. If $\theta<0,(a, a)$ is uniquely selected as $c \stackrel{g(\theta)}{\longrightarrow} b$ and $b \stackrel{g(\theta)}{\longrightarrow} a$.

\begin{tabular}{|c|c|c|c|}
\hline \multirow{4}{*}{ player $1 \stackrel{a}{b}$} & \multicolumn{3}{|c|}{ player 2} \\
\hline & $a$ & $b$ & $c$ \\
\hline & $4-\theta$ & $-\theta$ & $-4-\theta$ \\
\hline & 2 & 2 & 0 \\
\hline c & $\theta-6$ & $\theta$ & $2+\theta$ \\
\hline
\end{tabular}

FiguRE 6. Symmetric two player three action game

As indicated, theorem 9 allows the application of more general criteria. For instance, FMP find that symmetric binary action games are noise independent and give the following simple criterion to determine which action profile will be selected. Let $I=\{1, \ldots, I\}$, $A_{i \in I}=\{0,1\}$ and suppose that $g_{i \in I}\left(a_{i}, a_{-i}\right)$ depends only on $a_{i}$ and the number of opponents that play 1 (this is always true if payoffs are symmetric). Furthermore, let $\Delta^{n}$ denote the payoff difference on playing 1 rather than zero if $n$ opponents play 1 . Then $(1)_{i \in I}$ is the unique noise independent selection if $\sum_{n=0}^{I-1} \Delta^{n}>0$ and $(0)_{i \in I}$ is the uniquely noise independent selection if $\sum_{n=0}^{I-1} \Delta^{n}<0$. In other words, the global game approach selects the best reply on the conjecture that the number of opponents using action 1 is uniformly distributed between 0 and $|I|-1$. Theorem 9 allows us to apply this criterion to games with more than two actions.

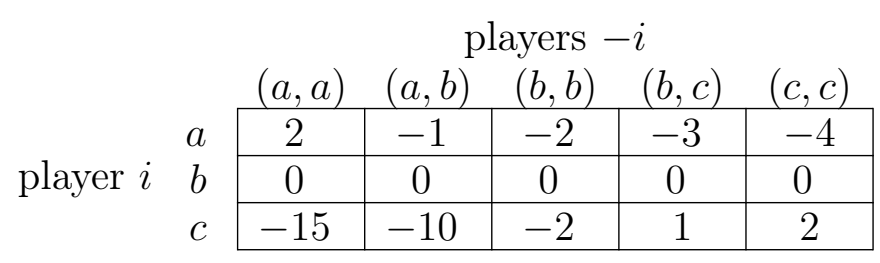

Figure 7. Symmetric three player game

Consider the three player, symmetric payoff game $g$ in figure 7 . As $b$ is a best reply if one expects his opponents to play $(a, a),(a, b)$ or $(b, b)$ with equal probability, we find that $b$ is the unique noise independent selection in $g \uparrow_{[a, b]}$, so $a \stackrel{g}{\rightarrow} b$. Analogously we find 
$b \stackrel{g}{\rightarrow} c$, so $a \stackrel{g}{\longmapsto} c$. Then theorem 9 implies that $(c, c, c)$ is selected uniquely and noise independently by the global game approach. Note that this result holds irrespective of the payoffs against $(a, c)$. Also, we could set the payoffs of playing $c$ versus $(a, a)$ or $(a, b)$ arbitrarily low without deterring players to play $c$.

\section{§4.3. Two player, $2 \times n$ action games}

Obviously, in order to fruitfully apply theorem 9, we need as many basic conditions as possible that guarantee noise independent selection for restricted games. Just counting the number of players and actions is certainly one of the most simple conditions to check. One possible extension of a two player $2 \times 2$ game is enlarging the action space of just one of the players. We will show that such games are always noise independent. Let $g$ be any game of strategic complementarities with $I=\{1,2\}, A_{1}=\{0,1\}, A_{2}=\left\{0,1, \ldots, m_{2}\right\}$. For example, figure 8 shows a $2 \times 3$ game. Note that this game does not have a $p$-dominant action profile.

\begin{tabular}{|c|c|c|c|}
\hline & & layer 2 & \\
\hline & 0 & 1 & 2 \\
\hline 10 & $0, \quad 4$ & $0, \quad 3$ & $0, \quad 0$ \\
\hline${ }^{1} 1$ & $\begin{array}{ll}0 & 0\end{array}$ & $\begin{array}{ll}0, & 3\end{array}$ & 1,5 \\
\hline
\end{tabular}

FiguRE 8 . A $2 \times 3$ game with no $p$-dominant action profile

For any $a_{2}^{*} \in A_{2}$, if $\left(0, a_{2}^{*}\right)$ is attained from below for some noise structure, $a_{2}^{*}$ is simply player 2's greatest best reply to player 1's action 0 , and can be attained from below under any noise structure. Now suppose the action profile $\left(1, a_{2}^{*}\right)$ is attained from below under $f$. Then there exists a lower- $f$-elaboration $\underline{e}(g, f)$ of $g$ with an equilibrium strategy profile $s^{*}$ that attains $\left(1, a_{2}^{*}\right)$, and $s^{*}$ may be identified with the thresholds $z_{1}^{0}$ and $z_{2}^{0}, z_{2}^{1}, \ldots, z_{2}^{k}$, where players switch to greater actions. Without loss of generality, we may assume all thresholds are below $R-\frac{1}{2}$ (since we can always increase $R$, extending the region of the signal space where players play the action profile $\left.\left(1, a_{2}^{*}\right)\right)$. The opposing action distribution faced by player 1 at $x_{1}=z_{1}^{0}$ is determined by the probabilities

$$
\mathbb{P}\left(x_{2}<z_{2}^{j} \mid x_{1}=z_{1}^{0}\right)=\int_{-\infty}^{z_{2}^{j}} \pi_{1}\left(x_{2} \mid z_{1}^{0}\right) d x_{2}, \quad j \in\{0, \ldots, k\} .
$$

The opposing action distribution that player 2 faces at each of her thresholds $z_{2}^{j}$ is

$$
\mathbb{P}\left(x_{1}>z_{1}^{0} \mid x_{2}=z_{2}^{j}\right)=\mathbb{P}\left(x_{2}<z_{2}^{j} \mid x_{1}=z_{1}^{0}\right):=p_{j}, \quad j \in\{0, \ldots, k\},
$$

where the equalities follow from lemma 1 stated in section 2.2.

Let $\underline{z}=\min \left\{z_{1}^{0}, z_{2}^{0}, z_{2}^{1}, \ldots, z_{2}^{k}\right\}$ be the smallest of the thresholds used in the strategy profile $s^{*}$, and let $i^{*}$ be the associated player switching at $\underline{z}$ (that is, $\underline{z}=z_{i^{*}}^{0}$ ). Now, if we consider a different noise structure $f^{\prime}$, we can construct a similarly increasing strategy profile $s^{* *}$ by again putting $i^{*}$ s smallest threshold $\tilde{z}_{i^{*}}^{0}$ to $\underline{z}$, and then simply rearranging 
the $k+1$ remaining thresholds $\left\{\tilde{z}_{1}^{0}, \tilde{z}_{2}^{0}, \tilde{z}_{2}^{1}, \ldots, \tilde{z}_{2}^{k}\right\}-\left\{\tilde{z}_{i^{*}}^{0}\right\}$ such that the $k+1$ equations

$$
\mathbb{P}\left(x_{2}<\tilde{z}_{2}^{0} \mid x_{1}=\tilde{z}_{1}^{0}\right)=p_{j}, \quad \text { for } j \in\{0, \ldots, k\}
$$

hold under the new noise structure. In this way, the action distributions of both players at all of their thresholds remains unchanged. Hence, since $s^{*}$ is an equilibrium strategy in $\underline{e}(g, f)$, it must be the case that $s^{* *}$ is an equilibrium strategy in $\underline{e}\left(g, f^{\prime}\right)$, and clearly $s^{* *}$ attains $\left(1, a_{2}^{*}\right)$. So $\left(1, a_{2}^{*}\right)$ is attained from below under $f^{\prime}$, and we may conclude that $\bar{a}\left(g, f^{\prime}\right) \geq \bar{a}(g, f)$. By a symmetric argument, $\bar{a}(g, f) \geq \bar{a}\left(g, f^{\prime}\right)$. Thus $\bar{a}(g, f)=\bar{a}\left(g, f^{\prime}\right)$, and by duality we may conclude that $\underline{a}(g, f)=\underline{a}\left(g, f^{\prime}\right)$. Since $f$ and $f^{\prime}$ were arbitrary, $g$ is noise independent. This establishes

Proposition 10. Any $2 \times n$ game of strategic complementarities is noise independent.

\section{§4.4. Examples of Noise Dependence}

For an example where theorem 9 is of no help, turn to the game in figure 9 . It is the 2 player, 4 action counterexample to noise independence that was discovered by FMP. Using the attainability criterion, we find that $b \stackrel{g}{\longmapsto} a$ and $b \stackrel{g}{\longmapsto} d$, so we lack a unique focal point. All we can say is that either $(a, a)$ or $(d, d)$ will be selected.

\begin{tabular}{ccc|c|c|c|} 
& \multicolumn{4}{c}{ player 2} \\
& & \multicolumn{1}{c}{$a$} & \multicolumn{1}{c}{$b$} & \multicolumn{1}{c}{$c$} \\
\cline { 3 - 6 } player & $a$ & 2000 & 1936 & 1144 & 391 \\
\cline { 3 - 6 } & $b$ & 1656 & 2000 & 1600 & 1245 \\
\cline { 3 - 6 } & $b$ & 1056 & 1800 & 2000 & 1660 \\
\cline { 3 - 6 } & $d$ & 254 & 1000 & 2160 & 2000 \\
\cline { 3 - 6 } & & & & &
\end{tabular}

Figure 9. FMP's counterexample

However, FMP's counterexample is not the smallest possible counterexample to noise independence. We conclude this section with two minimal examples. These examples show how the idea of attainability may be applied to establish the noise dependence of a game.

The first example is a noise dependent two player $3 \times 3$ game. This is a minimal counterexample to noise independence, since we have seen that all smaller two player games are noise independent. The second example is a noise dependent three player binary action game. It is minimal since all smaller binary action games are noise independent. As noted before, an analysis of this second type of game that establishes their noise dependence can already be found in Carlsson [3]. However, Carlsson's analysis does not fit well with the usual definition of a global game, since in his setup the players' signals are not conditionally independent, which is required to apply the theory of FMP. In our example this assumption is satisfied.

Let $g$ be the two player $3 \times 3$ game given by $I=\{1,2\}, A_{i \in I}=\{a, b, c\}$ and payoffs as in figure 10. First suppose $\eta_{1}$ is distributed uniformly over $\left[-\frac{1}{2}, \frac{1}{2}\right]$ while $\eta_{2}$ is distributed 


\begin{tabular}{|c|c|c|c|}
\hline & & player 2 & \\
\hline & $a$ & $b$ & $c$ \\
\hline & $30, \quad 10$ & $-15, \quad 0$ & $-15,-15$ \\
\hline player 1 & $0, \quad 0$ & $0, \quad 0$ & $0, \quad 0$ \\
\hline & $-10,-40$ & $-10, \quad 0$ & $10, \quad 10$ \\
\hline
\end{tabular}

Figure 10. Asymmetric two player three action game

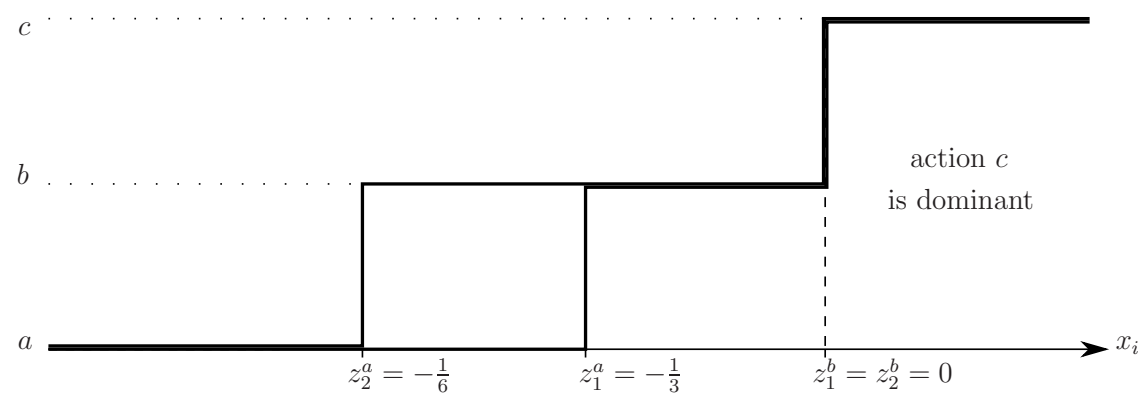

Figure 11. $(a, a)$ is attained from above under $f$ in $\bar{e}(g, f)$.

uniformly over $\left[-\frac{1}{10}, \frac{1}{10}\right]$. Then $(a, a)$ will be a global game selection. To see this, consider the upper- $f$-elaboration of $g$, in which the action $c$ is dominant for strictly positive signals. The state parameter $\theta$ is distributed uniformly over some interval $\left[L, \frac{1}{2}\right]$, and given a signal $x_{i} \in\left[L+\frac{1}{2}, 0\right]$ the conditional distribution that each player holds over his opponent's signal is given by the density

$$
\pi_{i}\left(x_{-i} \mid x_{i}\right)= \begin{cases}1, & 0 \leq\left|x_{-i}-x_{i}\right| \leq 0.4 \\ 3-5\left|x_{-i}-x_{i}\right|, & 0.4<\left|x_{-i}-x_{i}\right| \leq 0.6\end{cases}
$$

Now, consider the strategy profile in which player 1 switches to action $b$ at $z_{1}^{b}=0$ and to the lowest action $a$ at $z_{1}^{a}=-\frac{1}{6}$ - see figure 11. Player 2 switches at thresholds $z_{2}^{b}=0$ and $z_{2}^{a}=-\frac{1}{3}$.

If player 1 receives signal $x_{1}=0$, she assigns probabilities $\frac{1}{6}$, $\frac{1}{3}$ and $\frac{1}{2}$ to the events that his opponent chooses actions $a, b$ and $c$ respectively. Her expected payoff of playing $c$ is 0 and she is willing to switch from action $c$ to action $b$. Similarly, we find that $\mathbb{P}\left(x_{2} \leq z_{2}^{a} \mid x_{1}=z_{1}^{a}\right)=\mathbb{P}\left(z_{2}^{a}<x_{2} \leq z_{2}^{b} \mid x_{1}=z_{1}^{a}\right)=\mathbb{P}\left(x_{2}>z_{2}^{b} \mid x_{1}=z_{1}^{a}\right)=\frac{1}{3}$ and conclude that player 1 is willing to switch to the lowest action $a$ given a signal $x_{1}=z_{1}^{a}$.

For player 2 , we find that $\mathbb{P}\left(x_{1} \leq z_{1}^{a} \mid x_{2}=z_{2}^{b}\right)=\frac{1}{3}$ and $\mathbb{P}\left(x_{1}>z_{1}^{b} \mid x_{2}=z_{2}^{b}\right)=\frac{1}{2}$, implying that the expected payoff of playing $c$ is zero at $z_{2}^{b}$, hence equal to the payoff of playing $b$. Finally, we find that $\mathbb{P}\left(x_{1} \leq z_{1}^{a} \mid x_{2}=z_{2}^{a}\right)=\frac{2}{3}$ and $\mathbb{P}\left(x_{1}>z_{1}^{b} \mid x_{2}=z_{2}^{a}\right)=\frac{1}{6}$, implying that the expected payoff of playing $a$ given the signal $x_{2}=t_{2}^{a}$ is zero and equal to the payoff of playing $b$. Thus there exists an equilibrium profile in the upper- $f$-elaboration of $g$ in which the action profile $(a, a)$ is used, so the equilibrium $(a, a)$ of $g$ is selected by the left continuous version of the limit strategy profile.

However, if $\eta_{1}$ follows the density $f_{1}(x)=1-2 x$ with support $\left[-\frac{1}{2}, \frac{1}{2}\right]$, while $\eta_{2}$ is distributed uniformly over $[-\varepsilon, \varepsilon]$ the global game approach uniquely selects $(c, c)$ for sufficiently small $\varepsilon$. Consider a lower- $f$-elaboration of $g$ and a strategy profile specified 
by the four thresholds $z_{i}^{b}, z_{i}^{c}(i \in\{1,2\})$ at which the players switch to greater actions. Let $\Delta_{i}^{a, b}\left(x_{i}\right)$ denote player $i$ 's difference in payoff of playing $b$ rather than $a$ when given a signal $x_{i}$, and define $\Delta_{i}^{b, c}\left(x_{i}\right)$ analogously. We first examine the limit $\varepsilon=0$, so that player 2 is informed about the true state $\theta$. We find that for $z_{1}^{b}=0, z_{1}^{c}=0.15, z_{2}^{b}=0.07$, $z_{2}^{c}=0.34$

$$
\begin{gathered}
\Delta_{1}^{a, b}(0)=\frac{759}{2000} \approx 0.38, \quad \Delta_{1}^{b, c}(0.15)=\frac{239}{500} \approx 0.48 \\
\Delta_{2}^{a, b}(0.07)=\frac{61}{200} \approx 0.31, \quad \Delta_{2}^{b, c}(0.34)=\frac{69}{200} \approx 0.35
\end{gathered}
$$

so players strictly prefer to switch to greater actions at the thresholds and (conducting an upper-best reply iteration) we see that $(c, c)$ is attained from below under this noise structure. Indeed, since players strictly prefer to switch at the thresholds, the same must be true if we draw $x_{2}$ from $[\theta-\varepsilon, \theta+\varepsilon]$ and choose $\varepsilon$ very small - this only slightly perturbs expected payoffs. And even as we very slightly perturb the payoffs of the game $g$, the expected payoff differences at the thresholds remain positive. Thus $(c, c)$ is a global game selection for all games in some neighbourhood of the game $g$. This implies that $(c, c)$ is the unique noise independent global game selection in $g$ for both the left and right continuous

\begin{tabular}{|c|c|c|c|c|c|c|c|}
\hline & & & & & 1 and & play & \\
\hline & 0 & 1 & & $(0,0)$ & $(0,1)$ & $(1,0)$ & $(1,1)$ \\
\hline$\{1,2\}$ play & 0 & 0 & 3 & 0 & 0 & 0 & 0 \\
\hline$\imath \in\{1,2\}$ play, & -1 & 1 & 3 plays & -5 & 2 & 2 & 2 \\
\hline
\end{tabular}
versions of the limit strategy profile $s^{f}$.

FiguRE 12. Three player Soros game

Finally, consider the 3 player binary action game $g$ given by $I=\{1,2,3\}, A_{i \in I}=\{0,1\}$ and the payoffs given in figure 12. It is similar to the "Soros" game of Corsetti et al. [5]. We may think of player 3 as a large trader who can tip the balance, guaranteeing the success of a speculative attack, if she is joined by at least one of the small traders 1 or 2 . The game has two Nash equilibria, $(0,0,0)$ and $(1,1,1)$.

To determine which action profile will be selected by the global game approach, we turn to a lower- $f$-elaboration. Consider the noise structure $f$ such that for each $i \in I, \eta_{i}$ is distributed uniformly on $\left[-\frac{1}{2}, \frac{1}{2}\right]$. Then $(0,0,0)$ will be the global game selection. To see this, assume for the sake of argument that there is an equilibrium strategy profile where players 1 and 2 switch to action 1 at thresholds $z_{1}$ and $z_{2}$. As both players have the same payoff function and as $f_{1}=f_{2}$ we can assume without loss of generality that $z_{1}=z_{2}$. Moreover, as they are willing to switch at this threshold, they must assign a probability weakly greater than $\frac{1}{2}$ to the event that the third player plays 1 , i.e. they assume that player 3 switches to action 1 at some threshold $z_{3} \leq z_{1}=z_{2}$. Yet, if player 3 receives the critical signal $x_{3}^{*}=z_{1,2}$, she faces the opposing action profiles $(0,0),(0,1)$, and $(1,1)$ with equal probability (the "Laplacian" belief, see Morris and Shin [18]). So she cannot 
be willing to switch at $z_{3}$, as she would assign probability no less than $\frac{1}{3}$ to the event that opponents play $(0,0)$. We conclude that, under this noise structure, there can be no equilibrium strategy profile that attains $(1,1,1)$.

Now, consider the alternative noise structure $f^{\prime}$, in which $\eta_{1,2}^{\prime}$ are still uniformly distributed over $\left[-\frac{1}{2}, \frac{1}{2}\right]$, but $\eta_{3}^{\prime}$ is distributed over $[-\varepsilon, \varepsilon]$ for some $\varepsilon>0$. As $\varepsilon \rightarrow 0,(1,1,1)$ becomes the global game selection. For ease of presentation, let us assume that player 3 is informed about perfectly the true state $\theta$, viz $\varepsilon=0$; it will be clear from our argument that the result remains valid for small $\varepsilon$. Fix $z_{1}=z_{2}=z_{3}=0$. When receiving signal $x_{i}^{*}=0$, players 1 and 2 assign probability $\frac{1}{2}$ to the event that player 3 plays 1 , so they switch to action 1 at their threshold. Player 3 assigns probability $\frac{1}{4}$ to $(0,0)$ and to $(1,1)$, and probability $\frac{1}{2}$ to $(0,1)$. She, too, will switch to action 1 , since the expected payoff of playing 1 , which is $-5\left(\frac{1}{4}\right)+2\left(\frac{3}{4}\right)=\frac{1}{4}$, is strictly greater than the expected payoff of playing 0 , which yields 0 . Since this holds strictly, the argument remains valid if we draw her signal from $[-\varepsilon, \varepsilon]$ for sufficiently small $\varepsilon$.

\section{Robustness to Incomplete Information}

A lower(upper)-f-elaboration $\underline{e}(g, f)$ of $g$ is "close" to $g$ in the sense that conditional payoffs in $\underline{e}(g, f)$ coincide with the payoffs in $g$ with high ex ante probability. Kajii and Morris [15] examine incomplete information games that are close to some complete information game $g$ in this sense. Specifically, they look for a Nash equilibrium $a^{*}$ of $g$ for which, in every incomplete information game sufficiently close to $g$, there exists an equilibrium strategy profile $s$ in which players use the action profile $a^{*}$ with high probability. Such equilibria are called robust to incomplete information. We will show that if $a^{*}$ is robust to incomplete information, then $a^{*}$ is attained both from below and from above under any noise structure $f$. Thus, robustness to incomplete information implies noise independence. Morris and Ui [19] provide a large number of criteria that establish robustness to incomplete information. These conditions may be helpful for analysing global games, especially since the LP-maximiser condition in FMP — which was thought to guarantee noise independence - has recently been shown to be insufficient by Oyama and Takahashi [22].

The main conceptual problem that we need to solve in order to use all these known criteria for robustness to incomplete information is that Kajii and Morris only look at discrete incomplete information games.

Definition. A discrete incomplete information game $u$ consist of a finite player set $I$, finite action sets $A_{i \in I}$, a countable probability space $\Omega$ and state dependent payoff functions $u_{i}: A \times \Omega \rightarrow \mathbb{R}$. Each player receives a measurable signal $P_{i}(\omega)=p_{i}$, where $P_{i}$ can take on finitely many values and $\mathbb{P}\left(p_{i}\right)>0$ for each $p_{i} \in P_{i}[\Omega]$. Under these assumptions, the conditional probabilities $\mathbb{P}\left(\cdot \mid p_{i}\right)$ are well defined, so that players have well defined posteriors over the true state $\omega$ and their payoff function $u_{i}(\cdot, \omega)$. 
Let $\triangle\left(A_{i}\right)$ denote the set of all probability measures on $A_{i}$. A (mixed) strategy for player $i$ is a function $\sigma_{i}: P_{i}[\Omega] \rightarrow \triangle\left(A_{i}\right)$. When player $i$ uses the strategy $\sigma_{i}$, the probability that she chooses action $a_{i}$ given the signal $p_{i}$ is denoted by $\sigma_{i}\left(a_{i} \mid p_{i}\right)$. A strategy profile $\sigma=\left(\sigma_{i}\right)_{i}$ is a tuple of mixed strategies. The probability that the action profile $a=\left(a_{i}\right)_{i \in I}$ is played given the strategy profile $\sigma$ and given $\omega$ is denoted by $\sigma(a \mid \omega)$. The domain of $u_{i}$ extends to mixed strategies as follows:

$$
u_{i}(\sigma(\omega), \omega)=\sum_{a \in A} u_{i}(a, \omega) \sigma(a \mid \omega)
$$

A strategy profile is a (Bayes-Nash) equilibrium strategy profile of a discrete incomplete information game $u$ if for all $i \in I, p_{i} \in P_{i}(\Omega)$ and $a_{i} \in A_{i}$

$$
\sum_{\omega \in P^{-1}\left[\left\{p_{i}\right\}\right]} u_{i}(\sigma(\omega), \omega) \mathbb{P}\left(\omega \mid p_{i}\right) \geq \sum_{\omega \in P^{-1}\left[\left\{p_{i}\right\}\right]} u_{i}\left(a_{i}, \sigma_{-i}(\omega), \omega\right) \mathbb{P}\left(\omega \mid p_{i}\right)
$$

i.e. if it is a best reply to follow $\sigma_{i}$ on any signal $p_{i}$ against the opposing action distribution induced by $\sigma_{-i}$.

Now let $g$ be a complete information game of strategic complementarities. For an incomplete information game $u$, we define

$$
\Omega_{g}=\left\{\omega \mid u_{i}\left(\cdot, \omega^{\prime}\right)=g_{i}(\cdot) \text { for all } i \in I, \omega^{\prime} \in P_{i}^{-1}\left(P_{i}(\omega)\right)\right\}
$$

as the set of states where each player $i$ receives a signal $p_{i}$ telling her that her payoff function is $g_{i}$. A discrete incomplete information game $u$ said to be an $\varepsilon$-elaboration ${ }^{2}$ of $g$ if $\mathbb{P}\left(\Omega_{g}\right) \geq 1-\varepsilon$, following Kajii and Morris [15]. Although in the event $\Omega_{g}$ each player $i$ knows that payoffs are the same as in $g$, there will still be uncertainty about the signals that opponents receive, and this uncertainty affects higher order beliefs in $u$.

Definition. A Nash equilibrium $a^{*}$ of $g$ is said to be robust to incomplete information (Kajii and Morris [15]), or more succinctly, a robust equilibrium of $g$, if for every $\gamma>0$, there exists $\varepsilon>0$, such that in any $\varepsilon$-elaboration $u$ of $g$, there exists an equilibrium strategy profile $\sigma$ such that $a^{*}$ is played with ex ante probability at least $1-\gamma$, i.e. $\sum_{\omega \in \Omega} \sigma\left(a^{*} \mid \omega\right) \mathbb{P}(\omega) \geq 1-\gamma$.

The next theorem gives the formal link between robustness and noise independence, showing that every robust equilibrium is "sandwiched" between $\underline{s}^{f}$ and $\bar{s}^{f}$.

Theorem 11. Let $G_{v}(u, \phi, f)$ be any global game that embeds $g$ and $s^{f}$ its essentially unique limit strategy profile. Let $g=g\left(\theta^{*}\right)$. If $a^{*}$ is a robust equilibrium of $g$, then $\underline{s}^{f}\left(\theta^{*}\right) \leq a^{*} \leq \bar{s}^{f}\left(\theta^{*}\right)$, for any noise structure $f$.

This theorem slightly generalises a similar result by Oury and Tercieux [21], who use a more restrictive notion of robustness to incomplete information. They require that $a^{*}$ is robust not only in $g$ itself but in all complete information games in a neighbourhood of $g$, and then exploit a link with so-called "contagious" equilibria to show that their notion

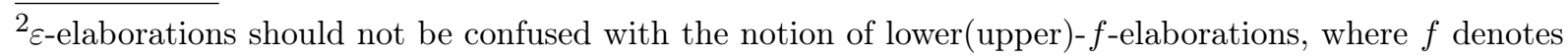
the noise structure instead of the size of the event $\Omega-\Omega_{g}$.
} 
implies that $a^{*}$ is the unique noise independent global game selection. We can connect our theorem to their result as follows:

Corollary 12. Let $G_{v}(u, \phi, f)$ be any global game and $s^{f}$ its essentially unique limit strategy profile. Suppose $g\left(\theta_{1}\right)=g$ and $g\left(\theta_{2}\right)=g^{\prime}$ for some $\theta_{1}<\theta_{2}$. If $a^{*}$ is a robust equilibrium of both $g$ and $g^{\prime}$, then it is the unique noise independent global game selection at any state parameter $\theta^{*} \in\left(\theta_{1}, \theta_{2}\right)$. More precisely, $\underline{s}^{f}\left(\theta^{*}\right)=a^{*}=\bar{s}^{f}\left(\theta^{*}\right)$ for any noise structure $f$.

Proof. Fix $f$ and some $\theta^{*} \in\left(\theta_{1}, \theta_{2}\right)$. Recall that the joint action space $A$ is finite. Since $s^{f}$ is increasing, it is discontinuous at finitely many points, implying

$$
\bar{s}^{f}\left(\theta_{1}\right) \leq \underline{s}^{f}\left(\theta^{*}\right) \leq \bar{s}^{f}\left(\theta^{*}\right) \leq \underline{s}^{f}\left(\theta_{2}\right) .
$$

From theorem 11 we infer $\underline{s}^{f}\left(\theta_{2}\right) \leq a^{*} \leq \bar{s}^{f}\left(\theta_{1}\right)$. Thus,

$$
\underline{s}^{f}\left(\theta^{*}\right)=a^{*}=\bar{s}^{f}\left(\theta^{*}\right) .
$$

We will prove theorem 11 in the obvious way, by showing that $a^{*}$ is attained from below (and dually, from above) under any noise structure $f$. So let us fix some $f$.

We start by discretising the information structure of lower- $f$-elaborations in order for them to fit the definition of a discrete incomplete information game. Let $\underline{e}(g, f)$ be a lower- $f$-elaboration of $g$ and, for $\delta>0$, let the signal space $\left[-\frac{1}{2}, R\right]$ of $\underline{e}(g, f)$ be covered by a partition of intervals of length $\delta$ :

$$
\begin{aligned}
& P^{\delta}=\left\{p^{n} \mid n \in\{\ell, \ell+1, \ldots, r\},\right\} \\
& \quad \text { where } p_{n}=[n \delta,(n+1) \delta), \\
& \quad \text { and } \ell, r \in \mathbb{Z}, \ell<r, \\
& \quad \text { and the partition } P^{\delta} \text { covers }\left[-\frac{1}{2}, R\right] .
\end{aligned}
$$

Now, for each $\delta>0$, we may consider a discrete incomplete information game $\underline{e}^{\delta}(g, f)$ based on $\underline{e}(g, f)$, in which instead of receiving their signal $x_{i}$, players are only informed about the interval $p \in P^{\delta}$ that contains $x_{i}$. A pure strategy profile $s$ in the $\underline{e}(g, f)$ is said to be an equilibrium under $\delta$-discretised information if and only if it is constant on every $p \in P^{\delta}$ and maximises expected payoff under this constraint, assuming that opponents follow the same strategy. More precisely:

$$
\forall i \forall a_{i} \forall p, \quad \int_{x_{i} \in p} u_{i}\left(s_{i}(p) \mid s, x_{i}\right) d x_{i} \geq \int_{x_{i} \in p} u_{i}\left(a_{i} \mid s, x_{i}\right) d x_{i},
$$

where, as before, $u_{i}\left(a_{i} \mid s, x_{i}\right)$ denotes the expected payoff of playing $a_{i} \in A_{i}$.

Theorem 11 is proved if we can show that some lower- $f$-elaboration $\underline{e}(g, f)$ has an equilibrium profile $s^{*}$ that attains $a^{*}$. The main step in our argument is given by the following lemma, which retrieves the robust equilibria of $g$ in our continuous lower- $f$-elaborations via the discrete incomplete information games $\underline{e}^{\delta}(g, f)$. 
Lemma 13. If $a^{*}$ is a robust equilibrium of $g$, then for any $\delta, 0<\delta<\frac{1}{2}$, there exists a lower-f-elaboration $\underline{e}(g, f)$ and a pure strategy profile $s^{*}$ in $\underline{e}(g, f)$ that is an equilibrium under $\delta$-discretised information and that attains $a^{*}$. Moreover, $s^{*}$ is increasing, right continuous, and is constant for signals greater than $2 R^{*}$.

Proof. Recall that in any lower- $f$-elaboration, the state parameter $\theta$ is distributed uniformly over some interval $\left[-\frac{1}{2}, R\right]$. For $\theta>\frac{1}{2}$, it is guaranteed that each player $i$ will receive a positive signal $x_{i}=\theta+\eta_{i}$ that informs her that the relevant individual payoff function $u_{i}\left(\cdot, x_{i}\right)$ is given by $g_{i}(\cdot)$. Now fix some $\delta, 0<\delta<\frac{1}{2}$. In any $\delta$-discretised lower-f-elaboration $\underline{e}^{\delta}(g, f)$, a realisation $\theta>\frac{1}{2}$ guarantees that each player $i$ knows that her payoff function is $g_{i}$, so

$$
\mathbb{P}\left(\Omega_{g}\right) \geq \mathbb{P}\left(\theta>\frac{1}{2}\right)=\frac{R-\frac{1}{2}}{R+\frac{1}{2}} .
$$

Since $a^{*}$ is a robust equilibrium of $g$, if we choose $R$ sufficiently large, there exists (by definition) a (mixed) equilibrium strategy profile $\sigma$ in $\underline{e}^{\delta}(g, f)$, such that $a^{*}$ is played in some interval $p^{*} \in P^{\delta}$ with strictly positive probability. So let us choose $R>2 R^{*}+2$ accordingly, thus fixing $\underline{e}^{\delta}(g, f)$.

Conducting an upper-best reply iteration in $\underline{e}^{\delta}(g, f)$ starting at $\sigma$ will give a pure equilibrium strategy profile $s$ that prescribes actions weakly greater than $a^{*}$ on the interval $p^{*}$.

Similarly, if we conduct an upper-best reply iteration in $\underline{e}^{\delta}(g, f)$ starting at

$$
\forall i \forall p, \quad s_{i}^{0}(p)=m_{i},
$$

we find the greatest (pure) strategy profile $s^{*} \geq s$ that is an equilibrium under $\delta$-discretised information (see figure 13). As $s^{*}$ is increasing, we can identify it with $k \leq R^{*}$ jump points $z_{1}, z_{2}, \ldots, z_{k}$, at each of which players switch to strictly greater actions. Under $\delta$ discretised information, each jump point $z_{n}$ influences the expected payoff on all intervals $p$ contained in $[z-1-2 \delta, z+1+2 \delta]$, so the maximum distance between any two adjacent jump points $z_{n}, z_{n+1}$ is $1+2 \delta<2$. Otherwise, the similarly increasing strategy profile given by the jump-points:

$$
z_{1}, z_{2}, \ldots, z_{n}-\delta, z_{n+1}-\delta, \ldots, z_{k}-\delta
$$

would also be an equilibrium, contradicting the maximality of $s^{*}$. Therefore, $s^{*} \geq s$ is constant for signals greater than $2 R^{*}$. If we interpret $s^{*}$ as an strategy in $\underline{e}(g, f)$, i.e.

$$
\text { for all } i, \quad s_{i}^{*}\left(x_{i}\right)=s_{i}^{*}\left(p^{k}\right) \text { for } x_{i} \in p^{k},
$$

it is an equilibrium under $\delta$-discretised information, right continuous and increasing, and satisfies $s^{*}(x) \geq a^{*}$ for $x \geq 2 R^{*}$.

Thus, if $a^{*}$ is a robust equilibrium, then for each sufficiently small $\delta$ there is some elaboration $\underline{e}(g, f)$ of $g$ with a strategy profile $s$ that attains $a^{*}$ such that $s$ is an equilibrium strategy profile under $\delta$-discretised information. As we choose $\delta$ smaller and smaller, the discretised information structures start resembling the continuous information structures 


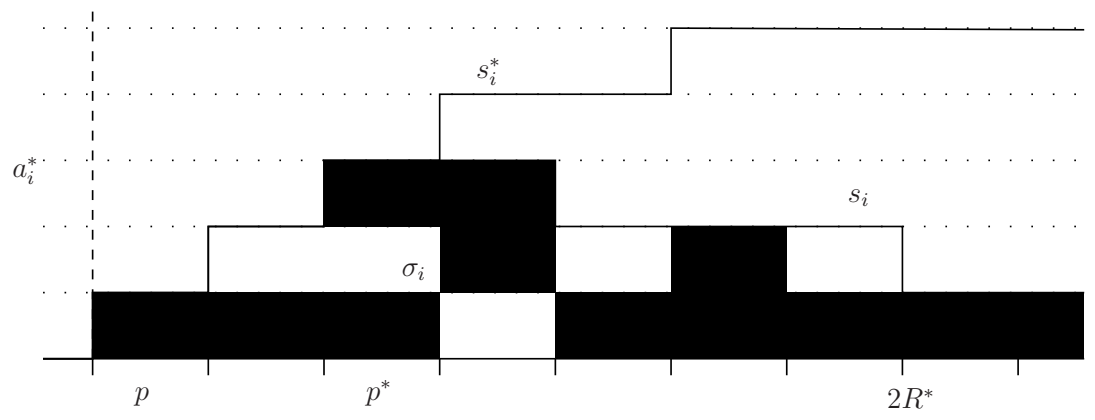

FiguRE 13. Robustness implies existence of a mixed strategy profile $\sigma$ that prescribes $a^{*}$ with positive probability. An upper-best reply iteration from the mixed strategy profile $\sigma$ yields the pure strategy profile $s$. The greatest equilibrium $s^{*} \geq s$ is constant for signals greater than $2 R^{*}$.

ever more closely. Intuitively, there should be an equilibrium strategy profile in some lower- $f$-elaboration $\underline{e}(g, f)$ that attains $a^{*}$, which is all we need to show. To take this final step, we need two new concepts.

Definition. Consider the lower-f-elaboration $\underline{e}(g, f)$ with $\theta$ distributed over the interval $\left[-\frac{1}{2}, 2 R^{*}+2\right]$. Every right continuous, increasing strategy $s_{i}$ in $\underline{e}(g, f)$ can be represented as an $\left(m_{i}+1\right)$-tuple of jump points, viz. an element of

$$
\mathscr{M}_{i}:=\left\{s_{i}=\left(y_{i}^{0}, y_{i}^{1}, y_{i}^{2} \ldots, y_{i}^{m_{i}}\right) \in \mathbb{R}^{A_{i}} \mid-\frac{1}{2} \leq y_{i}^{0} \leq y_{i}^{1} \leq y_{i}^{2} \leq \cdots \leq y_{i}^{m_{i}} \leq 2 R^{*}+3\right\},
$$

where $y_{i}^{a_{i}}$ denotes the least signal at which player $i$ plays some action $a_{i}^{\prime} \geq a_{i}$. The joint strategy space $\mathscr{M}:=\prod_{i \in I} \mathscr{M}_{i}$ contains all such strategy profiles. We will be interested in the subset of strategy profiles which use actions weakly greater than $a^{*}$ for signals $x_{i} \geq 2 R^{*}$ and the least action 0 for signals $x_{i}<0$. We denote it by:

$$
\mathscr{M}_{a^{*}}:=\left\{\left(y_{i}^{0}, \ldots y_{i}^{m_{i}}\right)_{i \in I} \in \mathscr{M} \mid \forall i, y_{i}^{a_{i}^{*}} \leq 2 R^{*} \text {, and } \forall i \forall a_{i}, y_{i}^{a} \geq 0\right\} \text {. }
$$

Note that $\mathscr{M}_{a^{*}}$ is a closed subset of the compact set $\left[0,2 R^{*}+3\right]^{A}$, hence compact.

Definition. A player's relative loss function in $\underline{e}(g, f)$ is defined as

$$
r_{i}(s)\left(x_{i}\right):=u_{i}\left(\hat{\beta}(s)_{i}\left(x_{i}\right) \mid s, x_{i}\right)-u_{i}\left(s_{i}\left(x_{i}\right) \mid s, x_{i}\right) .
$$

Moreover, we define

$$
r_{i}(s):=\int_{\bar{X}_{i}} r_{i}(s)\left(x_{i}\right) d x_{i}
$$

where $\bar{X}_{i}=\left[L-\frac{1}{2}, 2 R^{*}+2+\frac{1}{2}\right]$ denotes the set of possible signals. Finally, we set

$$
r(s):=\max _{i \in I} r_{i}(s)
$$

refer to it as relative loss of the strategy profile $s$.

Intuitively, $r(s)$ measures the distance between a strategy profile and its best reply; if $r(s)=0, s_{i}\left(x_{i}\right)$ is a best reply to $s$ for almost all $x_{i}$, which means (after adjusting $s$ on a null set if necessary) that we have found an equilibrium strategy profile. 
By identifying increasing, right continuous strategies with elements of $\mathscr{M}$ we can regard $r$ as a function on $\mathscr{M}$. To prove theorem 11, it suffices to establish that $r(s)=0$ somewhere on the compact set $\mathscr{M}_{a^{*}}$. To this end, the next lemma establishes a crucial property of $r$.

Lemma 14. The function $r: \mathscr{M}_{a^{*}} \rightarrow \mathbb{R}$ is continuous.

Proof. We will first show that $r_{i}(s)\left(x_{i}\right)$ is continuous for each $x_{i}$. For each $s \in \mathscr{M}_{a^{*}}$ and for each player $i$, the payoff function is bounded, and the difference in payoff between playing $s\left(x_{i}\right)$ and playing a best reply at $x_{i}$ is 0 if $x_{i}<0$ and less than

$$
T_{i}:=\max \left\{g_{i}(a) \mid a \in A\right\}-\min \left\{g_{i}(a) \mid a \in A\right\} \geq u_{i}\left(\hat{\beta}(s)_{i}\left(x_{i}\right) \mid s, x_{i}\right)-u_{i}\left(s_{i}\left(x_{i}\right) \mid s, x_{i}\right) .
$$

otherwise. Let $s^{n}$ be an arbitrary sequence converging to $s$ in the (compact metric) space $\mathscr{M}_{a^{*}}$. Observe that convergence in $\mathscr{M}_{a^{*}}$ is equivalent to the $s^{n}$ converging to $s$ pointwise almost everywhere when the $s^{n}$ are regarded, as usual, as measurable functions on the space $\left[-\frac{1}{2}, 2 R+3\right]^{I}$. Thus, by the dominated convergence theorem, for any $i, x_{i}$, and $a_{i}$, $u_{i}\left(a_{i} \mid s^{n}, x_{i}\right)$ converges to $u_{i}\left(a_{i} \mid s, x_{i}\right)$. Hence, for each $i, x_{i}$ and $a_{i}, u_{i}$ is continuous on $\mathscr{M}_{a^{*}}$. Since $\mathscr{M}_{a^{*}}$ is compact, $u_{i}$ is continuous, and $A_{i}$ is finite, Berge's maximum theorem [1, p. 116] then implies that $v_{i}(s)\left(x_{i}\right)=u_{i}\left(\hat{\beta}(s)_{i}\left(x_{i}\right) \mid s, x_{i}\right)$ is continuous on $\mathscr{M}_{a^{*}}$ for all $i, x_{i}$. It follows that

$$
r_{i}(s)\left(x_{i}\right):=v_{i}(s)\left(x_{i}\right)-u_{i}\left(s_{i}\left(x_{i}\right) \mid s, x_{i}\right)=u_{i}\left(\hat{\beta}(s)_{i}\left(x_{i}\right) \mid s, x_{i}\right)-u_{i}\left(s_{i}\left(x_{i}\right) \mid s, x_{i}\right)
$$

is continuous for all $i, x_{i}$.

Next, to prove the lemma, again let $s^{n}$ be an arbitrary sequence converging to $s$ in $\mathscr{M}_{a^{*}}$. For each $i$, the continuity of $r_{i}$ at each $x_{i}$ implies that $r_{i}\left(s^{n}\right)$ converges pointwise $r_{i}(s)$. From the dominated convergence theorem we deduce that

$$
r_{i}\left(s^{n}\right):=\int_{\bar{X}_{i}} r_{i}\left(s^{n}\right)\left(x_{i}\right) d x_{i} \text { converges to } \int_{\bar{X}_{i}} r_{i}(s)\left(x_{i}\right) d x_{i}=: r_{i}(s) \leq 2 R^{*} T_{i} .
$$

Since the player set $I$ is finite, this shows that $r(s)=\max _{i \in I} r_{i}(s)$ is continuous.

We are now set to prove theorem 11 .

Proof. Consider again the lower- $f$-elaboration $\underline{e}(g, f)$ with $\theta$ distributed over the interval $\left[-\frac{1}{2}, 2 R^{*}+2\right]$ and the associated strategy space $\mathscr{M}_{a^{*}}$. As promised, we will show that there is a strategy profile $s^{*} \in \mathscr{M}_{a^{*}}$ such that $r\left(s^{*}\right)=0$. Since $r$ is continuous by lemma 14, $r$ has a minimum on the compact set $\mathscr{M}_{a^{*}}$. It suffices to show that there are strategy profiles in $\mathscr{M}_{a^{*}}$ which have arbitrarily small relative loss.

First we will prove the following claim.

Claim. For every $i \in I$ and every $\gamma>0$, there exists $\kappa_{i}>0$ such that for all $a_{i} \in A_{i}$, all $s \in \mathscr{M}_{a^{*}}$, and all $x_{i}^{\prime}, x_{i}^{\prime \prime} \in\left[0,2 R^{*}+1\right]$ we have that:

$$
\left|x_{i}^{\prime}-x_{i}^{\prime \prime}\right|<\kappa_{i} \Longrightarrow\left|u_{i}\left(a_{i} \mid s, x_{i}^{\prime}\right)-u_{i}\left(a_{i} \mid s, x_{i}^{\prime \prime}\right)\right|<\gamma .
$$

Proof of claim. Choose any $i$. Consider the density function $\pi_{i}\left(x_{-i} \mid x_{i}\right)$ of player $i$ 's opponents' signals, conditional on $x_{i}$, as given by lemma 1 . The induced probability 
measure is absolutely continuous with respect to the Lebesgue measure $\boldsymbol{\mu}$ (Bogachev, [2], theorem 2.5.7), in other words, for any $\alpha>0$ there exists $\varepsilon^{\alpha}>0$ such that

$$
\boldsymbol{\mu}\left(D_{-i}\right) \leq \varepsilon^{\alpha} \Longrightarrow \int_{D_{-i}} \pi_{i}\left(x_{-i} \mid x_{i}\right) d x_{-i} \leq \alpha .
$$

Since changes in $x_{i}$ shift the probability density function $\pi_{i}\left(x_{-i} \mid x_{i}\right)$ in the real space but do not change its shape, $\alpha^{\varepsilon}$ can be chosen independently of $x_{i}$.

Now let $\gamma, s$, and $a_{i}$ be given, and assume without loss of generality that $k:=x_{i}^{\prime \prime}-x_{i}^{\prime}>0$. Consider the strategy profile $s^{\prime}$ defined by:

$$
\text { for all } i, \quad s_{i}^{\prime}\left(x_{i}\right)= \begin{cases}s_{i}\left(x_{i}\right) & \text { if } x_{i}<x_{i}^{\prime}-1 \\ s_{i}\left(x_{i}+k\right) & \text { if } x_{i}^{\prime}-1 \leq x_{i} \leq x_{i}^{\prime}+1 \\ s_{i}\left(x_{i}\right) & \text { if } x_{i}>x_{i}^{\prime}+1\end{cases}
$$

The strategy profiles $s_{-i}$ and $s_{-i}^{\prime}$ differ on a set $D_{-i}$. Clearly, for any $\varepsilon^{\alpha}$, there exists $\kappa_{i}>0$ sufficiently small to guarantee that $\left|x_{i}^{\prime \prime}-x_{i}^{\prime}\right|<\kappa_{i}$ implies $\boldsymbol{\mu}\left(D_{-i}\right) \leq \varepsilon^{\alpha}$. Choose $\alpha<\gamma / T_{i}$, choose $\varepsilon^{\alpha}$ in line with (1), and then $\kappa_{i}$ accordingly. We have:

$$
\begin{aligned}
& \left|u_{i}\left(a_{i} \mid s, x_{i}^{\prime}\right)-u_{i}\left(u_{i} \mid s, x_{i}^{\prime \prime}\right)\right| \\
& \quad \leq \int_{\bar{X}_{-i}}\left|g_{i}\left(a_{i}, s_{-i}\left(x_{-i}\right)\right) \pi_{i}\left(x_{-i} \mid x_{i}^{\prime}\right)-g_{i}\left(a_{i}, s_{-i}\left(x_{-i}\right)\right) \pi_{i}\left(x_{-i} \mid x_{i}^{\prime \prime}\right)\right| d x_{-i} \\
& \quad=\int_{\bar{X}_{-i}} \mid g_{i}\left(a_{i}, s_{-i}\left(x_{-i}\right) \pi_{i}\left(x_{-i} \mid x_{i}^{\prime}\right)-g_{i}\left(a_{i}, s_{-i}^{\prime}\left(x_{-i}\right) \pi_{i}\left(x_{-i} \mid x_{i}^{\prime}\right) \mid d x_{-i}\right.\right. \\
& \quad \leq \int_{D_{-i}} T_{i} \pi_{i}\left(x_{-i} \mid x_{i}^{\prime}\right) d x_{-i} \leq T_{i} \alpha<T_{i} \frac{\gamma}{T_{i}}=\gamma
\end{aligned}
$$

and thus the claim is proved.

Now, to prove the theorem, choose any $\varepsilon>0$, however small. We will find a strategy $s \in \mathscr{M}_{a^{*}}$ such that $r(s) \leq \varepsilon$. To this end, put $\gamma=\varepsilon /\left(8 R^{*}\right)$ and subsequently, for each $i \in I$, choose $\kappa_{i}<\frac{1}{2}$ according to our claim above. Let $\delta=\min _{i \in I} \kappa_{i}$.

Lemma 13 states that there exists a strategy profile $s^{\delta}$ that is an equilibrium under $\delta$-discretised information in some lower- $f$-elaboration of $g$, and that $s^{\delta}$ attains $a^{*}$, and is constant for signals above $2 R^{*}$. By restricting $s^{\delta}$ to the signal space of $\underline{e}(g, f)$, we can interpret $s^{\delta}$ as a strategy profile of $\underline{e}(g, f)$.

For any $i \in I$ and all signals $x_{i} \geq 2 R^{*}+1$, player $i$ knows that her opponents receive signals $x_{j} \geq 2 R^{*}$. Since $s^{\delta}$ is constant and equal to some $a \geq a^{*}$ for signals greater than $2 R^{*}$, the relative loss function $r_{i}\left(s^{\delta}\right)\left(x_{i}\right)$ is constant for $x_{i} \geq 2 R^{*}+1$. Moreover, since $s^{\delta}$ is an equilibrium under discretised information, we infer $r_{i}\left(s^{\delta}\right)\left(x_{i}\right)=0$ for $x_{i} \geq 2 R^{*}+1$. Similarly, for signals $x_{i}<0$, we have $r_{i}\left(s^{\delta}\right)\left(x_{i}\right)=0$, since $s_{i}^{\delta}\left(x_{i}\right)$ is equal to the least action 0 , the dominant action at such signals.

We turn to signals $x_{i} \in\left[0,2 R^{*}+1\right]$. On any interval $p \in P^{\delta}, s^{\delta}$ is constant and equal to some $a$ depending on $p$. For any pair of signals $x_{i}^{\prime}, x_{i}^{\prime \prime} \in p$ we have $\left|x_{i}^{\prime}-x_{i}^{\prime \prime}\right| \leq \delta \leq \kappa_{i}$, and hence our claim above implies that the expected payoff $u_{i}\left(a_{i} \mid s^{\delta}, x_{i}\right)$ from playing $s_{i}^{\delta}=a_{i}$ varies by less than $\gamma$ on the interval $p$. Now, if $u_{i}\left(a_{i} \mid s^{\delta}, x_{i}\right)$ varies by less than $\gamma$ on any 
interval $p \in P^{\delta}$, then $r_{i}\left(s^{\delta}\right)\left(x_{i}\right)$ varies by less than $2 \gamma$ on any interval $p$. To see this, assume the contrary, i.e. that for some $p \in P^{\delta}$ we could find $x_{i}^{\prime}, x_{i}^{\prime \prime} \in p$ such that

$$
r_{i}\left(s^{\delta}\right)\left(x_{i}^{\prime \prime}\right)-r_{i}\left(s^{\delta}\right)\left(x_{i}^{\prime}\right)>2 \gamma,
$$

that is,

$$
u_{i}\left(\hat{\beta}\left(s^{\delta}\right)_{i}\left(x_{i}^{\prime \prime}\right) \mid s^{\delta}, x_{i}^{\prime \prime}\right)-u_{i}\left(s_{i}^{\delta}\left(x_{i}^{\prime \prime}\right) \mid s^{\delta}, x_{i}^{\prime \prime}\right)-u_{i}\left(\hat{\beta}\left(s^{\delta}\right)_{i}\left(x_{i}^{\prime}\right) \mid s^{\delta}, x_{i}^{\prime}\right)+u_{i}\left(s_{i}^{\delta}\left(x_{i}^{\prime}\right) \mid s^{\delta}, x_{i}^{\prime}\right)>2 \gamma .
$$

Since $s_{i}^{\delta}\left(x_{i}^{\prime \prime}\right)=s_{i}^{\delta}\left(x_{i}^{\prime}\right)$, our claim above would then imply that

$$
\left|u_{i}\left(s_{i}^{\delta}\left(x_{i}^{\prime}\right) \mid s^{\delta}, x_{i}^{\prime}\right)-u_{i}\left(s_{i}^{\delta}\left(x_{i}^{\prime \prime}\right) \mid s^{\delta}, x_{i}^{\prime \prime}\right)\right|<\gamma,
$$

so that we could deduce

$$
u_{i}\left(\hat{\beta}\left(s^{\delta}\right)_{i}\left(x_{i}^{\prime \prime}\right) \mid s^{\delta}, x_{i}^{\prime \prime}\right)-\gamma>u_{i}\left(\hat{\beta}\left(s^{\delta}\right)_{i}\left(x_{i}^{\prime}\right) \mid s^{\delta}, x_{i}^{\prime}\right) .
$$

Furthermore, if at the signal $x_{i}^{\prime}$ player $i$ would play $\hat{\beta}\left(s^{\delta}\right)_{i}\left(x_{i}^{\prime \prime}\right)$, by our claim she would be able to guarantee herself a payoff within $\gamma$ of $u_{i}\left(\hat{\beta}\left(s^{\delta}\right)_{i}\left(x_{i}^{\prime \prime}\right) \mid s^{\delta}, x_{i}^{\prime \prime}\right)$ :

$$
u_{i}\left(\hat{\beta}\left(s^{\delta}\right)_{i}\left(x_{i}^{\prime \prime}\right) \mid s^{\delta}, x_{i}^{\prime}\right)>u_{i}\left(\hat{\beta}\left(s^{\delta}\right)_{i}\left(x_{i}^{\prime \prime}\right) \mid s^{\delta}, x_{i}^{\prime \prime}\right)-\gamma .
$$

So we infer $u_{i}\left(\hat{\beta}\left(s^{\delta}\right)_{i}\left(x_{i}^{\prime \prime}\right) \mid s^{\delta}, x_{i}^{\prime}\right)>u_{i}\left(\hat{\beta}\left(s^{\delta}\right)_{i}\left(x_{i}^{\prime}\right) \mid s^{\delta}, x_{i}^{\prime}\right)$, contradicting that $\hat{\beta}\left(s^{\delta}\right)_{i}\left(x_{i}^{\prime}\right)$ is a best reply.

Thus $r_{i}\left(s^{\delta}\right)\left(x_{i}\right)$ indeed varies by less than $2 \gamma$ over any interval $p \in P^{\delta}$. Moreover, since $s^{\delta}$ is an equilibrium under $\delta$-discretised information, we find that

$$
\forall i \forall p, \quad \int_{p} r_{i}\left(s^{\delta}\right)\left(x_{i}\right) d x_{i} \leq \delta 2 \gamma .
$$

Otherwise, putting $s_{i}^{\delta}(p)$ to some action that is a best reply at some $x_{i} \in p$, would yield a relative loss of less than $2 \gamma$ at all signals $x_{i} \in p$, which contradicts that $s^{\delta}$ minimises the average relative loss over $p$.

In sum,

$$
\begin{aligned}
\forall i, \quad r_{i}\left(s^{\delta}\right) & =\int_{\bar{X}_{i}} r_{i}\left(s^{\delta}\right)\left(x_{i}\right) d x_{i} \\
& =\int_{\left[0,2 R^{*}+1\right]} r_{i}\left(s^{\delta}\right)\left(x_{i}\right) d x_{i} \\
& \leq\left(2 R^{*}+1+\delta\right) 2 \gamma \leq\left(2 R^{*}+1+\delta\right) \frac{\varepsilon}{4 R^{*}}<\varepsilon .
\end{aligned}
$$

\section{Appendix}

Proof of Lemma 1. First, note that $x_{i} \in\left[L+\frac{1}{2}, R-\frac{1}{2}\right]$ implies

$$
\int_{[L, R]} f_{i}\left(\frac{x_{i}-\theta}{v}\right) d \theta=\int_{\left[x_{i}-\frac{v}{2}, x_{1}+\frac{v}{2}\right]} f_{i}\left(\frac{x_{i}-\theta}{v}\right) d \theta=1 .
$$


Thus,

$$
\begin{aligned}
f_{X_{i}=x_{i}}\left(\theta^{*}\right) & =\frac{f_{i}\left(\frac{x_{i}-\theta^{*}}{v}\right) \phi\left(\theta^{*}\right)}{\int_{\mathbb{R}} f_{i}\left(\frac{x_{i}-\theta}{v}\right) \phi(\theta) d \theta} \\
& =\frac{f_{i}\left(\frac{x_{i}-\theta^{*}}{v}\right) \frac{1}{R+L}}{\frac{1}{R+L} \int_{[L, R]} f_{i}\left(\frac{x_{i}-\theta}{v}\right) d \theta} \\
& =f_{i}\left(\frac{x_{i}-\theta^{*}}{v}\right),
\end{aligned}
$$

from which we conclude that

$$
\begin{aligned}
\pi_{i}\left(x_{-i} \mid x_{i}\right) & =\int_{[L, R]} \pi_{-i}\left(x_{-i} \mid \theta\right) f_{X_{i}=x_{i}}(\theta) d \theta \\
& =\int_{[L, R]} \prod_{i \in I} f_{i}\left(\frac{x_{i}-\theta}{v}\right) d \theta \\
& =\pi_{j}\left(x_{-j} \mid x_{j}\right) .
\end{aligned}
$$

\section{References}

[1] Berge, C. Topological Spaces: Including a Treatment of Multi-Valued Functions, Vector Spaces and Convexity. Mineola, New York: Dover Publications Inc, 1997.

[2] Bogachev, V. I. Measure Theory, vol. 1. Berlin, Heidelberg, New York: Springer, 2007.

[3] Carlsson, H. Global games and the risk dominance criterion. Working paper, University of Lund, 1989.

[4] Carlsson, H., And van Damme, E. Global games and equilibrium selection. Econometrica 61, 5 (1993), 989-1018.

[5] Corsetti, G., Dasgupta, A., Morris, S., And Shin, H. S. Does one Soros make a difference? a theory of currency crises with large and small traders. Review of Economic Studies 71, 1 (2004), 87-113.

[6] Corsetti, G., Guimarães, B., And Roubini, N. International lending of last resort and moral hazard: A model of IMF's catalytic finance. Journal of Monetary Economics 53, 3 (April 2006), 441-71.

[7] Cukierman, A., Goldstein, I., And Spiegel, Y. The choice of exchange-rate regime and speculative attacks. Journal of the European Economic Association 2, 6 (2004), 1206-41.

[8] Davey, B. A., And Priestley, H. A. Introduction to Lattices and Order, 2nd ed. Cambridge: Cambridge University Press, 2002.

[9] Diamond, D. W., And Dybvig, P. H. Bank runs, deposit insurance, and liquidity. Journal of Political Economy 91, 3 (1983), 401-19.

[10] Frankel, D. M., Morris, S., And Pauzner, A. Equilibrium selection in global games with strategic complementarities. Journal of Economic Theory 108, 1 (2003), 1-44.

[11] Goldstein, I. Strategic complementarities and the twin crises. The Economic Journal 115, 503 (2005), 368-90.

[12] Guimaraes, B., And Morris, S. Risk and wealth in a model of self-fulfilling currency attacks. Journal of Monetary Economics 54, 8 (2007), 2205-30.

[13] Heinemann, F., Nagel, R., And Ockenfels, P. The theory of global games on test: Experimental analysis of coordination games with public and private information. Econometrica 72, 5 (2004), 1583-99. 
[14] Heinemann, F., Nagel, R., and Ockenfels, P. Measuring strategic uncertainty in coordination games. Review of Economic Studies 76, 1 (2009), 181-221.

[15] Kajis, A., And Morris, S. The robustness of equilibria to incomplete information. Econometrica 65, 6 (1997), 1283-309.

[16] Morris, S., Rob, R., And Shin, H. S. p-dominance and belief potential. Econometrica 63, 1 (1995), 145-57.

[17] Morris, S., And Shin, H. S. Unique equilibrium in a model of self-fulfilling currency attacks. American Economic Review 88, 3 (1998), 587-97.

[18] Morris, S., And Shin, H. S. Global games: Theory and applications. In Advances in Economics and Econometrics: Eight World Congress, M. Dewatripont, L. P. Hansen, and S. J. Turnovsky, Eds. Cambridge: Cambridge University Press, 2003, pp. 56-114.

[19] Morris, S., AND Ui, T. Generalized potentials and robust sets of equilibria. Journal of Economic Theory 124, 1 (2005), 45-78.

[20] Obstfeld, M. Models of currency crises with self-fulfilling features. European Economic Review 40, 3-5 (1996), 1037-47.

[21] Oury, M., And Tercieux, O. Contagion in games with strategic complementarities. Manuscript, 2007.

[22] Oyama, D., And Takahashi, S. Monotone and local potential maximizers in symmetric $3 \times 3$ supermodular games. Economics Bulletin 29, 3 (2009), 2132-44.

[23] Oyama, D., And Tercieux, O. Iterated potential and robustness of equilibria. Journal of Economic Theory 144, 4 (2009), 1726-1769.

[24] Ray, D. What's new in development economics? The American Economist 44, 2 (2000), 3-16.

[25] Rochet, J.-C., And Vives, X. Coordination failures and the lender of last resort: Was Bagehot right after all? Journal of the European Economic Association 2, 6 (2004), 1116-47.

[26] TopkIs, D. M. Equilibrium points in nonzero-sum $n$-person submodular games. SIAM Journal on Control and Optimization 17, 6 (1979), 773-87.

[27] Topkis, D. M. Supermodularity and Complementarity. Frontiers of Economic Research. Princeton: Princeton University Press, 1998.

[28] Vives, X. Nash equilibrium with strategic complementarities. Journal of Mathematical Economics 19, 3 (1990), 305-21. 


\section{SFB 649 Discussion Paper Series 2010}

For a complete list of Discussion Papers published by the SFB 649, please visit http://sfb649. wiwi.hu-berlin.de.

001 "Volatility Investing with Variance Swaps" by Wolfgang Karl Härdle and Elena Silyakova, January 2010.

002 "Partial Linear Quantile Regression and Bootstrap Confidence Bands" by Wolfgang Karl Härdle, Ya'acov Ritov and Song Song, January 2010.

003 "Uniform confidence bands for pricing kernels" by Wolfgang Karl Härdle, Yarema Okhrin and Weining Wang, January 2010.

004 "Bayesian Inference in a Stochastic Volatility Nelson-Siegel Model" by Nikolaus Hautsch and Fuyu Yang, January 2010.

005 "The Impact of Macroeconomic News on Quote Adjustments, Noise, and Informational Volatility" by Nikolaus Hautsch, Dieter Hess and David Veredas, January 2010.

006 "Bayesian Estimation and Model Selection in the Generalised Stochastic Unit Root Model" by Fuyu Yang and Roberto Leon-Gonzalez, January 2010.

007 "Two-sided Certification: The market for Rating Agencies" by Erik R. Fasten and Dirk Hofmann, January 2010.

008 "Characterising Equilibrium Selection in Global Games with Strategic Complementarities" by Christian Basteck, Tijmen R. Daniels and Frank Heinemann, January 2010. 